\title{
Spectral form factors and late time quantum chaos
}

\author{
Junyu Liu ${ }^{*}$ \\ Walter Burke Institute for Theoretical Physics, California Institute of Technology, \\ Pasadena, California 91125, USA \\ and Institute for Quantum Information and Matter, California Institute of Technology, \\ Pasadena, California 91125, USA
}

(Received 27 July 2018; published 31 October 2018)

\begin{abstract}
This is a collection of notes about spectral form factors of standard ensembles in random matrix theory, written for the practical usage of the current study of late time quantum chaos. More precisely, we consider the Gaussian unitary ensemble, the Gaussian orthogonal ensemble, the Gaussian symplectic ensemble, the Wishart-Laguerre unitary ensemble, the Wishart-Laguerre orthogonal ensemble, and the Wishart-Laguerre symplectic ensemble. These results and their physics applications cover a threefold classification of late time quantum chaos in terms of spectral form factors.
\end{abstract}

DOI: $10.1103 /$ PhysRevD.98.086026

\section{OVERVIEW}

The theory of quantum chaos, and its connection to random matrix theory, have several new recent developments on understanding novel behaviors of condensed matter systems and the quantum nature of black hole physics. The definition of quantum chaos has various versions. Following the pioneering works done by Wigner [1] and Dyson [2], people regard random matrix theory as a tool to classify a generic random Hamiltonian with discrete symmetries, and their energy spectra have been observed to satisfy universal behaviors [3-5]. The scientific interest of random matrix theory varies from nonlinear science, mathematics, and mathematical physics, to nuclear physics, statistical physics, and quantum field theory. (See, for instance, [6-10] for reference.) Some recent discoveries about black hole physics have lead to interest in the understanding of scrambling properties of quantum chaotic systems [11-13], where people start to consider an early chaotic behavior. The Lyaponov exponent appears in the out-of-time-ordered correlators of the large $N$ theory [14,15], which is bounded by temperature in the thermal ensemble [16]. A concrete condensed matter model, the Sachedev-Ye-Kitaev (SYK) model [17-19], has been proposed to realize the chaotic properties that saturate the bound. Interestingly, one can also apply the random matrix theory classification to the SYK model

\footnotetext{
jiliu2@ caltech.edu
}

Published by the American Physical Society under the terms of the Creative Commons Attribution 4.0 International license. Further distribution of this work must maintain attribution to the author(s) and the published article's title, journal citation, and DOI. Funded by SCOAP.
[20,21]. Moreover, the spectral form factor, namely, the analytic-continuated partition function correlations in the SYK model, could be matched with the prediction of the spectral form factor in random matrix theory [22]. Some further investigations show that the spectral form factor is one of the key roles serving in several quantum chaotic systems, and it could connect to out-of-time-ordered correlators and some other chaotic diagnostics [23-25].

Those facts motivate us to study the spectral form factor in random matrix theory and its mathematical properties, in detail, from a modern chaotic physicist point of view. In this paper, we are mostly interested in the higher-point spectral form factors and how to reach them, in general, from some building blocks. As an explicit example, we will describe the four point spectral form factors, which are mostly closed to the four point out-of-time-ordered correlators.

From Dyson's classification, for Gaussian ensembles one could classify them by antiunitary symmetries as a Gaussian unitary ensemble (GUE), a Gaussian orthogonal ensemble (GOE), and a Gaussian symplectic ensemble (GSE). For real systems like the SYK model, those ensembles often appear periodically in a list from a number of sites. In this paper, we will consider all of them. Moreover, we will also discuss the Wishart-Laguerre ensembles with three symmetry classes. Those ensembles will correspond to supersymmetrized SYK models [26-28]. As some examples of physics applications, we will comment on SYK model classifications, out-of-timeordered-correlators, and Page states.

This paper is organized as follows. In Sec. II, we will discuss the spectral form factor in the GUE, the simplest symmetry class. In Sec. III, we will extend our discussions to the GOE and the GSE. In Sec. IV, we will discuss the spectral form factor properties of the Wishart-Laguerre ensembles. In Sec. V, we plot some figures for random matrix theory form factors to show their behaviors. 
In Sec. VI, we put the collections of physics applications about spectral form factors in random matrix theory. In Sec. VII, we will arrive at the conclusion and discussion.

\section{GUE SPECTRAL FORM FACTOR}

\section{A. Random matrix theory overview}

We consider the GUE in this section. This ensemble is defined by introducing the following distribution function over the space of Hermitian matrices $L \times L$,

$$
P(H) \propto \exp \left(-\frac{L}{2} \operatorname{Tr}\left(H^{2}\right)\right),
$$

which means that, for Hermitian matrices $H$, the offdiagonal elements are independent complex random distributions following Gaussian distribution with a mean of 0 and variance $1 / L$, while the diagonal elements are independent real random distributions following Gaussian distribution with a mean of 0 and variance $1 / L$. From this form, one can observe that the GUE ensemble is invariant under a unitary transformation $H \rightarrow U H U^{\dagger}$.

One can also write the result in the eigenvalue basis, where one can show that the distribution over a set of matrices could reduce to the distribution of eigenvalues with the following joint distribution,

$P\left(\lambda_{1}, \lambda_{2} \ldots, \lambda_{L}\right)=\exp \left(-\frac{L}{2} \sum_{i} \lambda_{i}^{2}\right) \prod_{i<j}\left(\lambda_{i}-\lambda_{j}\right)^{2}$

where $\lambda_{i} \mathrm{~s}$ are eigenvalues. We could write down the measure of it more formally by defining the Vandermonde determinant

$$
\Delta(\lambda)=\prod_{i<j}\left(\lambda_{i}-\lambda_{j}\right)
$$

and we could formally write down the measure

$$
P(\lambda) d \lambda=D \lambda=\exp \left(-\frac{L}{2} \sum_{i} \lambda_{i}^{2}\right) \Delta(\lambda)^{2} .
$$

Thus, based on this, we could compute the $n$-point correlation function, where $n<L$ as

$$
\rho^{(n)}\left(\lambda_{1}, \ldots, \lambda_{n}\right)=\int d \lambda_{n+1} \ldots d \lambda_{L} P\left(\lambda_{1}, \ldots, \lambda_{L}\right),
$$

where we are going to integrate out all eigenvalues from $n+1$ to $L$. One might be interested in what the result of the correlation function is if we take the large $L$ limit. From random matrix theory, people find that the $n$-point function could be determined by a kernel $K$

$$
\rho^{(n)}\left(\lambda_{1}, \ldots, \lambda_{n}\right)=\frac{(L-n) !}{L !} \operatorname{det}\left(K\left(\lambda_{i}, \lambda_{j}\right)\right)_{i, j=1}^{n},
$$

where the kernel $K$, in the large $L$ limit, behaves as

$$
K\left(\lambda_{i}, \lambda_{j}\right) \equiv \begin{cases}\frac{L}{\pi} \frac{\sin \left(L\left(\lambda_{i}-\lambda_{j}\right)\right)}{L\left(\lambda_{i}-\lambda_{j}\right)} & \text { for } i \neq j \\ \frac{L}{2 \pi} \sqrt{4-\lambda_{i}^{2}} & \text { for } i=j\end{cases}
$$

The kernel packages information about random matrix theory in the large $L$ limit, where, at the colliding case $i=j$, this kernel, as a one-point function, serves as Wigner's semicircle law. While in the case where $i \neq j$, this kernel is called the sine kernel in random matrix theory, which is universal even in most standard ensembles beyond the GUE.

The main goal of this paper is to try to build up the technology on how to compute the Fourier transformation of the $n$-point correlation functions, which is called the spectral form factor,

$$
\mathcal{R}_{2 k}(t)=\sum_{i, j} \int D \lambda e^{i\left(\lambda_{i_{1}}+\cdots+\lambda_{i_{k}}-\lambda_{j_{1}}-\cdots-\lambda_{j_{k}}\right)},
$$

where $k$ is any positive integer. We will start from our simplest example, the two-point form factor

$$
\mathcal{R}_{2}(t)=\sum_{i, j} \int D \lambda e^{i\left(\lambda_{i}-\lambda_{j}\right)},
$$

and we will discuss how to compute higher points and finite temperature results.

\section{B. Two-point form factor}

\section{The disconnected piece}

We start to compute the two-point form factor $\mathcal{R}_{2}$,

$$
\begin{aligned}
\mathcal{R}_{2}(t) & =\sum_{i, j} \int d \lambda_{i} d \lambda_{j} \rho^{(2)}\left(\lambda_{i}, \lambda_{j}\right) e^{i\left(\lambda_{i}-\lambda_{j}\right) t} \\
& =L+L(L-1) \int d \lambda_{1} d \lambda_{2} \rho^{(2)}\left(\lambda_{1}, \lambda_{2}\right) e^{i\left(\lambda_{1}-\lambda_{2}\right) t} .
\end{aligned}
$$

By directly computing the determinant, we have

$$
\begin{aligned}
\rho^{(2)}\left(\lambda_{1}, \lambda_{2}\right)= & \frac{L^{2}}{L(L-1)} \rho\left(\lambda_{1}\right) \rho\left(\lambda_{2}\right) \\
& -\frac{L^{2}}{L(L-1)} \frac{\sin ^{2}\left(L\left(\lambda_{1}-\lambda_{2}\right)\right)}{\left(L \pi\left(\lambda_{1}-\lambda_{2}\right)\right)^{2}},
\end{aligned}
$$

where we define 


$$
\rho(\lambda) \equiv \rho^{(1)}(\lambda),
$$

which has been reduced to the Wigner semicircle

$$
\rho(\lambda) \equiv \rho^{(1)}(\lambda)=\frac{1}{2 \pi} \sqrt{4-\lambda^{2}} .
$$

We call the leading piece disconnected, and it is relatively simple to deal with it. The Fourier transformation along this part is

$\mathcal{R}_{2}^{\text {disc }}(t)=L^{2} \int d \lambda_{1} d \lambda_{2} \rho\left(\lambda_{1}\right) \rho\left(\lambda_{2}\right) e^{i\left(\lambda_{1}-\lambda_{2}\right) t}=L^{2} r_{1}^{2}(t)$,

where the function $r_{1}(t)$ is written as

$$
r_{1}(t)=\frac{J_{1}(2 t)}{t}
$$

where $J_{\nu}(z)$ means the standard notation of the Bessel function.

\section{The connected piece: box approximation}

Now let us discuss the connected piece, which is defined as

$$
\begin{aligned}
\mathcal{R}_{2}^{\text {conn }}(t) & =\mathcal{R}_{2}(t)-\mathcal{R}_{2}^{\text {disc }}(t) \\
& =L-L^{2} \int d \lambda_{1} d \lambda_{2} \frac{\sin ^{2}\left(L\left(\lambda_{1}-\lambda_{2}\right)\right)}{\left(L \pi\left(\lambda_{1}-\lambda_{2}\right)\right)^{2}} e^{i\left(\lambda_{1}-\lambda_{2}\right) t} .
\end{aligned}
$$

However, the integral that appearing here, is divergent. The reason is that the sine kernel written here cannot probe two energy eigenvalues, $\lambda_{1}$ and $\lambda_{2}$, that are very close to each other, more precisely, around $\left|\lambda_{1}-\lambda_{2}\right| \sim 1 / L$. However, we could invent a technology that is called a box approximation that could still capture some physics, which we will describe as the following.

First, try to do a coordinate transformation

$$
\begin{aligned}
& u_{1}=\lambda_{1}-\lambda_{2} \\
& u_{2}=\lambda_{2},
\end{aligned}
$$

and thus, the integral becomes

$$
\begin{gathered}
L^{2} \int d \lambda_{1} d \lambda_{2} \frac{\sin ^{2}\left(L\left(\lambda_{1}-\lambda_{2}\right)\right)}{\left(L \pi\left(\lambda_{1}-\lambda_{2}\right)\right)^{2}} e^{i\left(\lambda_{1}-\lambda_{2}\right) t} \\
=L^{2} \int d u_{1} d u_{2} \frac{\sin ^{2}\left(L u_{1}\right)}{L \pi u_{1}^{2}} e^{i u_{1} t} .
\end{gathered}
$$

The expression, written in this form, manifests the divergence because we have an uncontrolled integral over the variable $u_{2}$. Now, let us first integrate over the variable $u_{1}$. Implementing the integral, we have

$$
L^{2} \int d u_{1} \frac{\sin ^{2}\left(L u_{1}\right)}{\left(L \pi u_{1}\right)^{2}} e^{i u_{1} t}=\frac{L}{\pi} \begin{cases}1-\frac{t}{2 L} & \text { for } t<2 L \\ 0 & \text { for } t>2 L\end{cases}
$$

so the whole connected piece should be given by this function times the volume of the integration region of $u_{2}$ : $\operatorname{vol}(\mathbb{R})$. However, one could try to cut off the integration range by brute force to get a finite value. Let us assume that this cutoff space is symmetric around the origin, [-cut, cut], then the result is given by

$$
\begin{gathered}
L^{2} \int d \lambda_{1} d \lambda_{2} \frac{\sin ^{2}\left(L\left(\lambda_{1}-\lambda_{2}\right)\right)}{\left(L \pi\left(\lambda_{1}-\lambda_{2}\right)\right)^{2}} e^{i\left(\lambda_{1}-\lambda_{2}\right) t} \\
=\frac{2 \operatorname{cut} \times L}{\pi} \begin{cases}1-\frac{t}{2 L} & \text { for } t<2 L \\
0 & \text { for } t>2 L .\end{cases}
\end{gathered}
$$

One can try to solve the cut by checking the consistency of the result at $t=0$. At $t=0$, we know that the disconnected piece has contributed $L^{2}$, which is the whole form factor result, so the connected piece should obtain zero at $t=0$, which means that

$$
2 \mathrm{cut}=\operatorname{vol}(\mathbb{R})=\pi \rightarrow \mathrm{cut}=\frac{\pi}{2} .
$$

One can see, which we will discuss later, that this cutoff $\pi / 2$ also works for higher-point cases. Thinking about the origin of it, we first write down the one-point function with the Wigner semicircle law

$$
\rho(\lambda)=\frac{1}{2 \pi} \sqrt{4-\lambda^{2}} .
$$

Now, let us pretend that $\lambda$ is very small, which is close to the origin, and then we have

$$
\rho(\lambda=0)=\frac{1}{\pi} .
$$

Now, we could approximate, for a small enough $\lambda$, that the semicircle distribution is approximately a line. To compute the length of this line, we could use the normalization condition. The integral over $\rho$ is normalized by 1 , so if we choose our line to be distributed in the range [-cut, cut], we get $2 \mathrm{cut} / \pi=1$, namely, cut $=\pi / 2$. A short explanation of this phenomena is that the box approximation is a brute force choice to make up the difference between the sine kernel and the semicircle when two energies are very close to each other $\lambda_{1} \rightarrow \lambda_{2}$.

There is another interpretation to this result. The connected part of the two-point form factor is a linear increase, from $(0,0)$ to $(2 L, L)$ in the coordinate $\left(t, \mathcal{R}_{2}^{\text {conn }}(t)\right)$, and it 
stops growing (we call it as plateau). The origin $(0,0)$ is fixed, and the plateau time, $t_{p}=2 L$, is fixed by the property of the Fourier transformation of the sine kernel, which will be independent of the cutoff choice. The plateau value, $\left.\mathcal{R}_{2}^{\text {conn }}\left(t_{p}=2 L\right)\right)=L$, is fixed by the long time average interpretation of the definition of the form factor (which means that the damping $e^{\left(i\left(\lambda_{1}-\lambda_{2}\right) t\right)}$ for $\lambda_{1} \neq \lambda_{2}$ will be canceled after long time averaging, and the only constant piece with $\lambda_{1}=\lambda_{2}$ will give the result $L$ because there are $L$ eigenvalues in total). Thus, drawing a line from $(0,0)$ to $(2 L, L)$, assuming linearity, has to obtain the slope $1 / 2$. Because $(2 L, L)$ is already fixed, we could claim that the result beyond the box approximation should be some nonlinear physics.

As a summary, we obtain the connected piece of the twopoint form factor, given by

$$
\mathcal{R}_{2}^{\text {conn }}=L\left(1-r_{2}(t)\right),
$$

where $r_{2}(t)$ is defined as

$$
r_{2}(t)= \begin{cases}1-\frac{t}{2 L} & \text { for } t<2 L \\ 0 & \text { for } t>2 L\end{cases}
$$

\section{The connected piece: an improvement}

Now, we introduce an improvement that is more refined than the box cutoff. In this part, we will try to use the short distance kernel,

$$
\tilde{K}\left(\lambda_{i}, \lambda_{j}\right)=L \frac{\sin \left(\pi L\left(\lambda_{i}-\lambda_{j}\right) \rho\left(\left(\lambda_{i}+\lambda_{j}\right) / 2\right)\right)}{\pi L\left(\lambda_{i}-\lambda_{j}\right)},
$$

where this kernel is an approximation when $\lambda_{i}$ and $\lambda_{j}$ are sufficiently close. The following technology is also mentioned in [24], but the results here, as far as we know, are novel.

Take this kernel in our hand, let us try to compute the connected part of the form factor. It is now captured by an integral,

$L^{2} \int d \lambda_{1} d \lambda_{2} \frac{\sin ^{2}\left(\pi L\left(\lambda_{1}-\lambda_{2}\right) \rho\left(\left(\lambda_{1}+\lambda_{2}\right) / 2\right)\right)}{\left(\pi L\left(\lambda_{i}-\lambda_{j}\right)\right)^{2}} e^{i\left(\lambda_{1}-\lambda_{2}\right) t}$.

Here, we try applying a different coordinate transformation

$$
\begin{aligned}
& u_{1}=\lambda_{1}-\lambda_{2} \\
& u_{2}=\frac{\lambda_{1}+\lambda_{2}}{2},
\end{aligned}
$$

so the integral becomes

$$
\begin{aligned}
& L^{2} \int d \lambda_{1} d \lambda_{2} \frac{\sin ^{2}\left(\pi L\left(\lambda_{1}-\lambda_{2}\right) \rho\left(\left(\lambda_{1}+\lambda_{2}\right) / 2\right)\right)}{\left(\pi L\left(\lambda_{i}-\lambda_{j}\right)\right)^{2}} e^{i\left(\lambda_{1}-\lambda_{2}\right) t} \\
& \quad=L^{2} \int d u_{1} d u_{2} \frac{\sin ^{2}\left(\pi L u_{1} \rho\left(u_{2}\right)\right)}{\left(\pi L u_{1}\right)^{2}} e^{i u_{1} t} .
\end{aligned}
$$

With the treatment here, we could split the space of $u_{1}$ in $\mathbb{R}$ by an infinite number of intervals $\Omega$ at the center $u_{2}$, with the assumption that the integrand outside of the interval will quickly decay. Suppose that we are now at the center, $u_{2}$, and the interval has the range $\left[-\Omega_{0} / 2, \Omega_{0} / 2\right]$, then by implementing the integral, in the large $L$ limit, we have

$$
\begin{aligned}
L^{2} & \int_{-\Omega_{0} / 2}^{\Omega_{0} / 2} d u_{1} \frac{\sin ^{2}\left(\pi L u_{1} \rho\left(u_{2}\right)\right)}{\left(\pi L u_{1}\right)^{2}} e^{i u_{1} t} \\
& =\frac{L}{\pi} \rho\left(u_{2}\right) \int_{-L \rho\left(u_{2}\right) \pi \Omega_{0} / 2}^{L \rho\left(u_{2}\right) \pi \Omega_{0} / 2} d u_{1} \frac{\sin ^{2}\left(u_{1}\right)}{u_{1}^{2}} e^{i u_{1} t / L \pi \rho\left(u_{2}\right)} \\
& \sim \frac{L}{\pi} \rho\left(u_{2}\right) \int_{-\infty}^{+\infty} d u_{1} \frac{\sin ^{2}\left(u_{1}\right)}{u_{1}^{2}} e^{i u_{1} t / L \pi \rho\left(u_{2}\right)} \\
& =L \rho\left(u_{2}\right) \begin{cases}1-\frac{t}{2 \pi L \rho\left(u_{2}\right)} & \text { for } t<2 \pi L \rho\left(u_{2}\right) \\
0 & \text { for } t>2 \pi L \rho\left(u_{2}\right)\end{cases} \\
& =\max \left(L \rho\left(u_{2}\right)-\frac{t}{2 \pi}, 0\right) .
\end{aligned}
$$

Here, an assumption that we are making is that we are extending the range from an $L$ amplified interval to infinity, regardless of the fact that the exponent will be $\mathcal{O}(1)$ even if $u_{1}$ could scale as $\mathcal{O}(L)$.

Now, we sum over all of the intervals, which means that we are integrating over $u_{2}$ in the range $[-2,2]$ (the range of the semicircle), and we get

$$
\begin{aligned}
& L^{2} \int d u_{1} d u_{2} \frac{\sin ^{2}\left(\pi L u_{1} \rho\left(u_{2}\right)\right)}{\left(\pi L u_{1}\right)^{2}} e^{i u_{1} t} \\
& =\int_{-2}^{2} d u_{2} \max \left(L \rho\left(u_{2}\right)-\frac{t}{2 \pi}, 0\right) \\
& = \begin{cases}\frac{2}{\pi} L \operatorname{arccsc}\left(\frac{2 L}{\sqrt{4 L^{2}-t^{2}}}\right)-\frac{t}{2 \pi L} \sqrt{4 L^{2}-t^{2}} & \text { for } t<2 L \\
0 & \text { for } t>2 L .\end{cases}
\end{aligned}
$$

Thus, the connected form factor is given by

$$
\mathcal{R}_{2}^{\operatorname{conn}}(t)= \begin{cases}L-\frac{2}{\pi} L \operatorname{arccsc}\left(\frac{2 L}{\sqrt{4 L^{2}-t^{2}}}\right)+\frac{t}{2 \pi L} \sqrt{4 L^{2}-t^{2}} & \text { for } t<2 L \\ L & \text { for } t>2 L .\end{cases}
$$


This result will capture more accurate physics. One interesting thing is that in the early time, we expand it in small $t$, and we get

$$
\mathcal{R}_{2}^{\mathrm{conn}}(t)=\frac{2 t}{\pi}-\frac{t^{3}}{12 \pi L^{2}}-\frac{t^{5}}{320 \pi L^{4}}+\mathcal{O}\left(t^{6}\right)
$$

Thus, this method will give the slope $2 / \pi$ in the early time. This fact is verified by numerics in [23], but with the plateau still $(2 L, L)$. The reason is that the function in the middle is nonlinear. One can estimate the nonlinear time scale, which is given by $t=\mathcal{O}(L)$, where in this time scale the higher-order corrections to the linear function become important.

However, as this refined technology cannot be generalized simply to a higher-point case, we will keep using the box cutoff for a higher-point case, which is believable for physics in spectral form factors.

\section{Higher point form factor: theorem}

Higher point form factor calculations are based on multivariable Fourier transformations of the determinant of sine kernels. We will derive some generic results to establish the framework of computing higher-point form factors, in general, based on the box approximation, and we compute a four-point example. Our starting point will be the following theorem,

Theorem 2.1 (Convolution formula for infinite $L$, in Eq. 5.2.23, [7]): We have the following formula to compute the convolution of the sine kernel:

$$
\begin{aligned}
\int & \prod_{i=1}^{m} d y_{i} \exp \left(2 \pi i \sum_{j=1}^{m} k_{j} y_{j}\right) s\left(y_{1}-y_{2}\right) \\
& \times s\left(y_{2}-y_{3}\right) \ldots s\left(y_{m-1}-y_{m}\right) s\left(y_{m}-y_{1}\right) \\
= & \delta\left(\sum_{j=1}^{m} k_{j}\right) \int d k g(k) g\left(k+k_{1}\right) \ldots g\left(k+k_{m-1}\right) .
\end{aligned}
$$

where $s$ is the sine kernel,

$$
s(r):=\frac{\sin (\pi r)}{\pi r}
$$

and the principle valued Fourier transformation of the sine kernel is given by

$$
\int e^{2 \pi i k r} s(r) d r=g(k)= \begin{cases}1 & |k|<\frac{1}{2} \\ 0 & |k|>\frac{1}{2}\end{cases}
$$

Proof.-Change the variables

$$
\begin{aligned}
u_{1} & =y_{1}-y_{2} \\
u_{2} & =y_{2}-y_{3} \\
& \ldots \\
u_{m-1} & =y_{m-1}-y_{m} \\
u_{m} & =y_{m},
\end{aligned}
$$

and the inverse transformation is

$$
\begin{aligned}
y_{1} & =u_{1}+u_{2}+u_{3}+\cdots+u_{m} \\
y_{2} & =u_{2}+u_{3}+\cdots+u_{m} \\
& \ldots \\
y_{m-2} & =u_{m-2}+u_{m-1}+u_{m} \\
y_{m-1} & =u_{m-1}+u_{m} \\
y_{m} & =u_{m},
\end{aligned}
$$

whose Jacobian is 1 . Thus, we obtain

$$
\begin{aligned}
& \int \prod_{i=1}^{m} d y_{i} \exp \left(2 \pi i \sum_{j=1}^{m} k_{j} y_{j}\right) s\left(y_{1}-y_{2}\right) s\left(y_{2}-y_{3}\right) \ldots s\left(y_{m-1}-y_{m}\right) s\left(y_{m}-y_{1}\right) \\
& =\int \prod_{i=1}^{m} d u_{i} \exp \left(2 \pi i \sum_{l=1}^{m} k_{l} \sum_{\alpha=j}^{m} u_{\alpha}\right) s\left(u_{1}\right) s\left(u_{2}\right) \ldots s\left(u_{m-1}\right) s\left(\sum_{j=1}^{m-1} u_{j}\right) \\
& =\int \prod_{i=1}^{m} d u_{i} \exp \left(2 \pi i \sum_{\alpha=1}^{m}\left(\sum_{l=1}^{\alpha} k_{l}\right) u_{\alpha}\right) s\left(u_{1}\right) s\left(u_{2}\right) \ldots s\left(u_{m-1}\right) s\left(\sum_{j=1}^{m-1} u_{j}\right) .
\end{aligned}
$$

From this, we observe that we could first read off the integral over $u_{m}$, which is 


$$
\begin{aligned}
& \int \prod_{i=1}^{m} d u_{i} \exp \left(2 \pi i \sum_{\alpha=1}^{m}\left(\sum_{l=1}^{\alpha} k_{l}\right) u_{\alpha}\right) s\left(u_{1}\right) s\left(u_{2}\right) \ldots s\left(u_{m-1}\right) s\left(\sum_{j=1}^{m-1} u_{j}\right) \\
& =\int \prod_{i=1}^{m-1} d u_{i} \exp \left(2 \pi i \sum_{\alpha=1}^{m-1}\left(\sum_{l=1}^{\alpha} k_{l}\right) u_{\alpha}\right) s\left(u_{1}\right) s\left(u_{2}\right) \ldots s\left(u_{m-1}\right) s\left(\sum_{j=1}^{m-1} u_{j}\right) \int \exp \left(2 \pi i\left(\sum_{l=1}^{m} k_{l}\right) u_{m}\right) d u_{m} \\
& =\delta\left(\sum_{l=1}^{m} k_{l}\right) \int \prod_{i=1}^{m-1} d u_{i} \exp \left(2 \pi i \sum_{\alpha=1}^{m-1}\left(\sum_{l=1}^{\alpha} k_{l}\right) u_{\alpha}\right) s\left(u_{1}\right) s\left(u_{2}\right) \ldots s\left(u_{m-1}\right) s\left(\sum_{j=1}^{m-1} u_{j}\right) .
\end{aligned}
$$

Now, to deal with the last sine kernel, introduce a new variable $u$, which is

$$
s\left(\sum_{j=1}^{m-1} u_{j}\right)=s\left(-\sum_{j=1}^{m-1} u_{j}\right)=\int d u s(u) \delta\left(u+\sum_{j=1}^{m-1} u_{j}\right),
$$

and then, replace the delta function by the exponential function

$$
s\left(\sum_{j=1}^{m-1} u_{j}\right)=\int d u d k s(u) \exp \left(2 \pi i k\left(u+\sum_{j=1}^{m-1} u_{j}\right)\right) .
$$

Inserting the integral, we have

$$
\begin{aligned}
& \int \prod_{i=1}^{m} d y_{i} \exp \left(2 \pi i \sum_{j=1}^{m} k_{j} y_{j}\right) s\left(y_{1}-y_{2}\right) s\left(y_{2}-y_{3}\right) \ldots s\left(y_{n-1}-y_{n}\right) s\left(y_{n}-y_{1}\right) \\
& =\delta\left(\sum_{l=1}^{m} k_{l}\right) \int \prod_{i=1}^{m-1} d u_{i} d u d k \exp \left(2 \pi i \sum_{\alpha=1}^{m-1}\left(\sum_{l=1}^{\alpha} k_{l}\right) u_{\alpha}\right) \exp \left(2 \pi i k\left(u+\sum_{j=1}^{m-1} u_{j}\right)\right) s\left(u_{1}\right) s\left(u_{2}\right) \ldots s\left(u_{m-1}\right) s(u) \\
& \left.=\delta\left(\sum_{l=1}^{m} k_{l}\right) \int \prod_{i=1}^{m-1} d u_{i} d u d k \exp \left(2 \pi i \sum_{\alpha=1}^{m-1}\left(\sum_{l=1}^{\alpha} k_{l}+k\right) u_{\alpha}\right) \exp (2 \pi i k u)\right) s\left(u_{1}\right) s\left(u_{2}\right) \ldots s\left(u_{m-1}\right) s(u) \\
& =\delta\left(\sum_{l=1}^{m} k_{l}\right) \int d k\left(\prod_{i=1}^{m-1} \int d u_{i} \exp \left(2 \pi i\left(\sum_{l=1}^{i} k_{l}+k\right) u_{i}\right) s\left(u_{i}\right)\right)\left(\int d u \exp (2 \pi i k u) s(u)\right) \\
& =\delta\left(\sum_{l=1}^{m} k_{l}\right) \int d k g(k) \prod_{i=1}^{m-1} g\left(\sum_{l=1}^{i} k_{l}+k\right)
\end{aligned}
$$

as desired.

Now, it is obvious to generalize this claim of a large but finite $L$. We have

$$
\begin{aligned}
& \int \prod_{i=1}^{m} d \lambda_{i} K\left(\lambda_{1}, \lambda_{2}\right) K\left(\lambda_{2}, \lambda_{3}\right) \ldots K\left(\lambda_{m-1}, \lambda_{m}\right) K\left(\lambda_{m}, \lambda_{1}\right) e^{i \sum_{i=1}^{m} k_{i} \lambda_{i}} \\
& =\frac{L}{\pi} \int d \lambda e^{i \sum_{i=1}^{m} k_{i} \lambda} \int d k g(k) g\left(k+\frac{k_{1}}{2 L}\right) g\left(k+\frac{k_{2}}{2 L}\right) \ldots g\left(k+\frac{k_{m-1}}{2 L}\right),
\end{aligned}
$$

where the delta function is replaced by an integral over the exponential function. We impose the box approximation again

$$
\int d \lambda e^{i \sum_{i=1}^{m} k_{i} \lambda} \rightarrow \int_{-\pi / 2}^{\pi / 2} d \lambda e^{i \sum_{i=1}^{m} k_{i} \lambda}
$$

which is always fixed by the normalization at the initial time, 


$$
\left.\frac{L}{\pi} \int_{-\pi / 2}^{\pi / 2} d \lambda e^{i \sum_{i=1}^{m} k_{i} \lambda} \int d k g(k) g\left(k+\frac{k_{1}}{2 L}\right) g\left(k+\frac{k_{2}}{2 L}\right) \ldots g\left(k+\frac{k_{m-1}}{2 L}\right)\right|_{k_{1}=k_{2}=\ldots k_{m-1}=0}=L,
$$

and we find the number $\pi / 2$ is universal for all $m$. We finally get the useful formula:

Theorem 2.2 (Convolution formula for finite large $L$ ):

$$
\begin{aligned}
& \int \prod_{i=1}^{m} d \lambda_{i} K\left(\lambda_{1}, \lambda_{2}\right) K\left(\lambda_{2}, \lambda_{3}\right) \ldots K\left(\lambda_{m-1}, \lambda_{m}\right) K\left(\lambda_{m}, \lambda_{1}\right) e^{i \sum_{i=1}^{m} k_{i} \lambda_{i}} \\
& =L r_{3}\left(\sum_{i=1}^{m} k_{i}\right) \int d k g(k) g\left(k+\frac{k_{1}}{2 L}\right) g\left(k+\frac{k_{2}}{2 L}\right) \ldots g\left(k+\frac{k_{m-1}}{2 L}\right),
\end{aligned}
$$

where we define the function

$$
r_{3}(t)=\frac{\sin (\pi t / 2)}{\pi t / 2}
$$

This convolution formula allows us to compute any higher-point spectral form factors. We will show an example about how the four-point form factor has been computed.

\section{Four-point form factor}

Now, let us consider the four-point form factor as an example

$$
\mathcal{R}_{4}=\sum_{a, b, c, d=1}^{L} \int D \lambda e^{i\left(\lambda_{a}+\lambda_{b}-\lambda_{c}-\lambda_{d}\right) t}
$$

Before our computation, we will define the following building block functions

$$
\begin{aligned}
& r_{1}(t)=\frac{J_{1}(2 t)}{t} \\
& r_{2}(t)= \begin{cases}1-\frac{t}{2 L} & \text { for } t<2 L \\
0 & \text { for } t>2 L\end{cases} \\
& r_{3}(t)=\frac{\sin (\pi t / 2)}{\pi t / 2} .
\end{aligned}
$$

Take a look at the classifications of combinations in $\mathcal{R}_{4}$, which are

(i) $a=b=c=d=e=f$ : Contribute $L$.

(ii) $a=b$ : Contribute $L(L-1)(L-2) \int D \lambda e^{i\left(2 \lambda_{1}-\lambda_{2}-\lambda_{3}\right) t}$.

(iii) $c=d$ : Contribute $L(L-1)(L-2) \int D \lambda e^{i\left(\lambda_{1}+\lambda_{2}-2 \lambda_{3}\right) t}$.

(iv) $a=c$ or $a=d$ or $b=c$ or $b=d$ : Contribute $4 L(L-1)(L-2) \int D \lambda e^{i\left(\lambda_{1}-\lambda_{2}\right) t}$.

(v) $b=c=d$ or $a=c=d$ or $a=b=d$ or $a=b=c$ : Contribute $4 L(L-1) \int D \lambda e^{i\left(\lambda_{1}-\lambda_{2}\right) t}$.

(vi) $a=b$ and $c=d$ : Contribute $L(L-1) \int D \lambda e^{i\left(2 \lambda_{1}-2 \lambda_{2}\right) t}$.

(vii) $a=c$ and $b=d$, or $a=d$ and $b=c$ : Contribute $2 L(L-1)$.

(viii) All inequal indexes: $L(L-1)(L-2)(L-3) \int D \lambda e^{i\left(\lambda_{1}+\lambda_{2}-\lambda_{3}-\lambda_{4}\right) t}$.

Adding the total prefactors will give $L^{4}$. Add them together and we get

$$
\begin{aligned}
\mathcal{R}_{4}= & L(L-1)(L-2)(L-3) \int D \lambda e^{i\left(\lambda_{1}+\lambda_{2}-\lambda_{3}-\lambda_{4}\right) t}+2 L(L-1)(L-2) \operatorname{Re} \int D \lambda e^{i\left(2 \lambda_{1}-\lambda_{2}-\lambda_{3}\right) t} \\
& +L(L-1) \int D \lambda e^{i\left(2 \lambda_{1}-2 \lambda_{2}\right) t}+4 L(L-1)^{2} \int D \lambda e^{i\left(\lambda_{1}-\lambda_{2}\right) t}+2 L^{2}-L \\
= & L(L-1)(L-2)(L-3) \int D \lambda e^{i\left(\lambda_{1}+\lambda_{2}-\lambda_{3}-\lambda_{4}\right) t}+2 L(L-1)(L-2) \operatorname{Re} \int D \lambda e^{i\left(2 \lambda_{1}-\lambda_{2}-\lambda_{3}\right) t} \\
& +L^{2}\left|r_{1}(2 t)\right|^{2}-L r_{2}(2 t)+4(L-1)\left(L^{2}\left|r_{1}(t)\right|^{2}-L r_{2}(t)\right)+2 L^{2}-L .
\end{aligned}
$$

We have already obtained what the last three terms are. Now we only need to consider the first two terms. 


\section{The first term}

The first term is an actual four-point function,

$$
L(L-1)(L-2)(L-3) \int D \lambda e^{i\left(\lambda_{1}+\lambda_{2}-\lambda_{3}-\lambda_{4}\right) t} .
$$

When expanding the determinant, the terms could be summarized as the following:

(i) 4-type: In this case, we have

$$
\begin{aligned}
& -2 \int d \lambda_{1} d \lambda_{2} d \lambda_{3} d \lambda_{4} K\left(\lambda_{1}, \lambda_{3}\right) K\left(\lambda_{3}, \lambda_{2}\right) K\left(\lambda_{2}, \lambda_{4}\right) K\left(\lambda_{4}, \lambda_{1}\right) e^{i\left(\lambda_{1}+\lambda_{2}-\lambda_{3}-\lambda_{4}\right) t} \\
& -2 \int d \lambda_{1} d \lambda_{2} d \lambda_{3} d \lambda_{4} K\left(\lambda_{1}, \lambda_{2}\right) K\left(\lambda_{2}, \lambda_{3}\right) K\left(\lambda_{3}, \lambda_{4}\right) K\left(\lambda_{4}, \lambda_{1}\right) e^{i\left(\lambda_{1}+\lambda_{2}-\lambda_{3}-\lambda_{4}\right) t} \\
& -2 \int d \lambda_{1} d \lambda_{2} d \lambda_{3} d \lambda_{4} K\left(\lambda_{1}, \lambda_{2}\right) K\left(\lambda_{2}, \lambda_{4}\right) K\left(\lambda_{4}, \lambda_{3}\right) K\left(\lambda_{3}, \lambda_{1}\right) e^{i\left(\lambda_{1}+\lambda_{2}-\lambda_{3}-\lambda_{4}\right) t},
\end{aligned}
$$

and thus the result is

$$
-6 L r_{2}(2 t)
$$

(ii) 1-1-1-1-type: In this case, we have

$$
\int d \lambda_{1} K\left(\lambda_{1}, \lambda_{1}\right) e^{i \lambda_{1} t} \int d \lambda_{2} K\left(\lambda_{2}, \lambda_{2}\right) e^{i \lambda_{2} t} \int d \lambda_{3} K\left(\lambda_{3}, \lambda_{3}\right) e^{-i \lambda_{3} t} \int d \lambda_{4} K\left(\lambda_{4}, \lambda_{4}\right) e^{-i \lambda_{4} t}
$$

This term contributes

$$
L^{4}\left|r_{1}(t)\right|^{4}
$$

(iii) 1-1-2-type: In this case, we have

$$
\begin{gathered}
-\int d \lambda_{1} d \lambda_{2} K^{2}\left(\lambda_{1}, \lambda_{2}\right) e^{i\left(\lambda_{1}+\lambda_{2}\right) t} \int d \lambda_{3} K\left(\lambda_{3}, \lambda_{3}\right) e^{-i \lambda_{3} t} \int d \lambda_{4} K\left(\lambda_{4}, \lambda_{4}\right) e^{-i \lambda_{4} t} \\
-\int d \lambda_{3} d \lambda_{4} K^{2}\left(\lambda_{3}, \lambda_{3}\right) e^{-i\left(\lambda_{3}+\lambda_{4}\right) t} \int d \lambda_{1} K\left(\lambda_{1}, \lambda_{1}\right) e^{i \lambda_{1} t} \int d \lambda_{2} K\left(\lambda_{2}, \lambda_{2}\right) e^{i \lambda_{2} t} \\
-\int d \lambda_{1} d \lambda_{4} K^{2}\left(\lambda_{1}, \lambda_{4}\right) e^{i\left(\lambda_{1}-\lambda_{4}\right) t} \int d \lambda_{2} K\left(\lambda_{2}, \lambda_{2}\right) e^{i \lambda_{2} t} \int d \lambda_{3} K\left(\lambda_{3}, \lambda_{3}\right) e^{-i \lambda_{3} t} \\
-\int d \lambda_{1} d \lambda_{3} K^{2}\left(\lambda_{1}, \lambda_{3}\right) e^{i\left(\lambda_{1}-\lambda_{3}\right) t} \int d \lambda_{2} K\left(\lambda_{2}, \lambda_{2}\right) e^{i \lambda_{2} t} \int d \lambda_{4} K\left(\lambda_{4}, \lambda_{4}\right) e^{-i \lambda_{4} t} \\
-\int d \lambda_{2} d \lambda_{4} K^{2}\left(\lambda_{2}, \lambda_{4}\right) e^{i\left(\lambda_{2}-\lambda_{4}\right) t} \int d \lambda_{1} K\left(\lambda_{1}, \lambda_{1}\right) e^{i \lambda_{1} t} \int d \lambda_{3} K\left(\lambda_{3}, \lambda_{3}\right) e^{-i \lambda_{3} t} \\
-\int d \lambda_{2} d \lambda_{3} K^{2}\left(\lambda_{2}, \lambda_{3}\right) e^{i\left(\lambda_{2}-\lambda_{3}\right) t} \int d \lambda_{1} K\left(\lambda_{1}, \lambda_{1}\right) e^{i \lambda_{1} t} \int d \lambda_{4} K\left(\lambda_{4}, \lambda_{4}\right) e^{-i \lambda_{4} t} .
\end{gathered}
$$

This term contributes

$$
-2 L^{3} \operatorname{Re}\left(r_{1}^{2}(t)\right) r_{2}(t) r_{3}(2 t)-4 L^{3}\left|r_{1}(t)\right|^{2} r_{2}(t) .
$$

(iv) 2-2-type: In this case, we have

$$
\begin{aligned}
& +\int d \lambda_{1} d \lambda_{4} K^{2}\left(\lambda_{1}, \lambda_{4}\right) e^{i\left(\lambda_{1}-\lambda_{4}\right) t} \int d \lambda_{2} d \lambda_{3} K^{2}\left(\lambda_{2}, \lambda_{3}\right) e^{i\left(\lambda_{2}-\lambda_{3}\right) t}+\int d \lambda_{1} d \lambda_{3} K^{2}\left(\lambda_{1}, \lambda_{3}\right) e^{i\left(\lambda_{1}-\lambda_{3}\right) t} \int d \lambda_{2} d \lambda_{4} K^{2}\left(\lambda_{2}, \lambda_{4}\right) e^{i\left(\lambda_{2}-\lambda_{4}\right) t} \\
& +\int d \lambda_{1} d \lambda_{2} K^{2}\left(\lambda_{1}, \lambda_{2}\right) e^{i\left(\lambda_{1}+\lambda_{2}\right) t} \int d \lambda_{3} d \lambda_{4} K^{2}\left(\lambda_{3}, \lambda_{4}\right) e^{-i\left(\lambda_{3}+\lambda_{4}\right) t}
\end{aligned}
$$


This term contributes

$$
2 L^{2} r_{2}^{2}(t)+L^{2} r_{2}^{2}(t) r_{3}^{2}(2 t)
$$

(v) 3-1-type: In this case, we have

$$
8 L^{2} \operatorname{Re}\left(r_{1}(t)\right) r_{2}(t) r_{3}(t)
$$

Finally, as a summary, we have

$$
\begin{aligned}
L(L-1)(L-2)(L-3) \int D \lambda e^{i\left(\lambda_{1}+\lambda_{2}-\lambda_{3}-\lambda_{4}\right) t}= & L^{4}\left|r_{1}(t)\right|^{4}-2 L^{3} \operatorname{Re}\left(r_{1}^{2}(t)\right) r_{2}(t) r_{3}(2 t)-4 L^{3}\left|r_{1}(t)\right|^{2} r_{2}(t) \\
& +2 L^{2} r_{2}^{2}(t)+L^{2} r_{2}^{2}(t) r_{3}^{2}(2 t)+8 L^{2} \operatorname{Re}\left(r_{1}(t)\right) r_{2}(t) r_{3}(t) \\
& -6 L r_{2}(2 t) .
\end{aligned}
$$

\section{The second term}

In this part, we will evaluate the second term,

$$
2 L(L-1)(L-2) \operatorname{Re} \int D \lambda e^{i\left(2 \lambda_{1}-\lambda_{2}-\lambda_{3}\right) t}
$$

Let us first consider it without a factor of 2 ,

$$
L(L-1)(L-2) \operatorname{Re} \int D \lambda e^{i\left(2 \lambda_{1}-\lambda_{2}-\lambda_{3}\right) t} .
$$

Then, we obtain:

(i) 3-type: In this case, we have

$$
2 \operatorname{Re} \int d \lambda_{1} d \lambda_{2} d \lambda_{3} K\left(\lambda_{1}, \lambda_{2}\right) K\left(\lambda_{2}, \lambda_{3}\right) K\left(\lambda_{3}, \lambda_{1}\right) e^{i\left(2 \lambda_{1}-\lambda_{2}-\lambda_{3}\right) t}
$$

This term contributes

$$
2 L r_{3}(t)
$$

(ii) 1-1-1-type: In this case, we have

$$
\operatorname{Re} \int d \lambda_{1} K\left(\lambda_{1}, \lambda_{1}\right) e^{2 i \lambda_{1} t} \int d \lambda_{2} K\left(\lambda_{2}, \lambda_{2}\right) e^{-i \lambda_{2} t} \int d \lambda_{3} K\left(\lambda_{3}, \lambda_{3}\right) e^{-i \lambda_{3} t}
$$

This term contributes

$$
L^{3} \operatorname{Re}\left(r_{1}(2 t) r_{1}^{* 2}(t)\right)
$$

(iii) 2-1-type: In this case, we have

$$
\begin{aligned}
& -\int d \lambda_{1} K\left(\lambda_{1}, \lambda_{1}\right) e^{i 2 \lambda_{1} t} \int d \lambda_{2} d \lambda_{3} K^{2}\left(\lambda_{2}, \lambda_{3}\right) e^{-i\left(\lambda_{2}+\lambda_{3}\right) t}-\int d \lambda_{2} K\left(\lambda_{2}, \lambda_{2}\right) e^{-i \lambda_{2} t} \int d \lambda_{1} d \lambda_{3} K^{2}\left(\lambda_{1}, \lambda_{3}\right) e^{i\left(2 \lambda_{1}-\lambda_{3}\right) t} \\
& -\int d \lambda_{3} K\left(\lambda_{3}, \lambda_{3}\right) e^{-i \lambda_{3} t} \int d \lambda_{1} d \lambda_{2} K^{2}\left(\lambda_{1}, \lambda_{2}\right) e^{i\left(2 \lambda_{1}-\lambda_{2}\right) t} .
\end{aligned}
$$

This term contributes

$$
-L^{2} \operatorname{Re}\left(r_{1}(2 t)\right) r_{3}(2 t) r_{2}(t)-2 L^{2} \operatorname{Re}\left(r_{1}^{*}(t)\right) r_{3}(t) r_{2}(2 t)
$$


So, finally, we make a summary that is

$$
\begin{aligned}
2 L(L-1)(L-2) \operatorname{Re} \int D \lambda e^{i\left(2 \lambda_{1}-\lambda_{2}-\lambda_{3}\right) t}= & 2 L^{3} \operatorname{Re}\left(r_{1}(2 t) r_{1}^{* 2}(t)\right)-2 L^{2} \operatorname{Re}\left(r_{1}(2 t)\right) r_{3}(2 t) r_{2}(t)-4 L^{2} \operatorname{Re}\left(r_{1}^{*}(t)\right) r_{3}(t) r_{2}(2 t) \\
& +4 L r_{2}(3 t)
\end{aligned}
$$

\section{The final result}

Here, we make a summary. The final result for $\mathcal{R}_{4}$ is

$$
\begin{aligned}
\mathcal{R}_{4}= & L^{4}\left|r_{1}(t)\right|^{4}-2 L^{3} \operatorname{Re}\left(r_{1}^{2}(t)\right) r_{2}(t) r_{3}(2 t)-4 L^{3}\left|r_{1}(t)\right|^{2} r_{2}(t)+2 L^{3} \operatorname{Re}\left(r_{1}(2 t) r_{1}^{* 2}(t)\right)+4 L^{3}\left|r_{1}(t)\right|^{2} \\
& +2 L^{2} r_{2}^{2}(t)+L^{2} r_{2}^{2}(t) r_{3}^{2}(2 t)+8 L^{2} \operatorname{Re}\left(r_{1}(t)\right) r_{2}(t) r_{3}(t)-2 L^{2} \operatorname{Re}\left(r_{1}(2 t)\right) r_{3}(2 t) r_{2}(t) \\
& -4 L^{2} \operatorname{Re}\left(r_{1}^{*}(t)\right) r_{3}(t) r_{2}(2 t)+L^{2}\left|r_{1}(2 t)\right|^{2}-4 L^{2}\left|r_{1}(t)\right|^{2}-4 L^{2} r_{2}(t)+2 L^{2} \\
& -7 L r_{2}(2 t)+4 L r_{2}(3 t)+4 L r_{2}(t)-L .
\end{aligned}
$$

In the large $L$ limit, one can find some terms are suppressed in the all time scale. We could also have an approximate formula

$$
\mathcal{R}_{4}=L^{4}\left|r_{1}(t)\right|^{4}+2 L^{2} r_{2}^{2}(t)-4 L^{2} r_{2}(t)+2 L^{2}-7 L r_{2}(2 t)+4 L r_{2}(3 t)+4 L r_{2}(t)-L,
$$

which captures the main physics of the four-point spectral form factor.

\section{E. Finite temperature result}

Finally, we will take a look at the finite temperature result, where this result will also rely on the refined kernel and the interval splitting technology, as mentioned before; here, we only precisely compute the two-point case. The definition of the finite temperature two-point form factor is

$$
\mathcal{R}_{2}=\sum_{i, j} \int D \lambda e^{i\left(\lambda_{i}-\lambda_{j}\right) t} e^{-\beta\left(\lambda_{i}-\lambda_{j}\right)}
$$

Following from a simple analysis, we have

$$
\begin{aligned}
\mathcal{R}_{2}=\sum_{i, j} \int D \lambda e^{i\left(\lambda_{i}-\lambda_{j}\right) t} e^{-\beta\left(\lambda_{i}+\lambda_{j}\right)} & =L \int d \lambda \rho(\lambda) e^{-2 \beta \lambda}+L(L-1) \int d \lambda_{1} d \lambda_{2} \rho^{(2)}(\lambda) e^{i\left(\lambda_{1}-\lambda_{2}\right) t} e^{-\beta\left(\lambda_{1}+\lambda_{2}\right)} \\
& =L r_{1}(2 i \beta)+L^{2} r_{1}(t+i \beta) r_{1}(t-i \beta)-\int d \lambda_{1} d \lambda_{2} K^{2}\left(\lambda_{1}, \lambda_{2}\right) e^{i\left(\lambda_{1}-\lambda_{2}\right) t} e^{-\beta\left(\lambda_{1}+\lambda_{2}\right),}
\end{aligned}
$$

so we have the separation

$$
\begin{aligned}
& \mathcal{R}_{2}^{\mathrm{disc}}(t, \beta)=L^{2} r_{1}(t+i \beta) r_{1}(t-i \beta) \\
& \mathcal{R}_{2}^{\text {conn }}(t, \beta)=L r_{1}(2 i \beta)-\int d \lambda_{1} d \lambda_{2} K^{2}\left(\lambda_{1}, \lambda_{2}\right) e^{i\left(\lambda_{1}-\lambda_{2}\right) t} e^{-\beta\left(\lambda_{1}+\lambda_{2}\right) .}
\end{aligned}
$$

Thus, for the connected part, we could compute the integral

$$
L^{2} \int d \lambda_{1} d \lambda_{2} \frac{\sin ^{2}\left(\pi L\left(\lambda_{1}-\lambda_{2}\right) \rho\left(\left(\lambda_{1}+\lambda_{2}\right) / 2\right)\right)}{\left(\pi L\left(\lambda_{i}-\lambda_{j}\right)\right)^{2}} e^{i\left(\lambda_{1}-\lambda_{2}\right) t} e^{-\beta\left(\lambda_{1}+\lambda_{2}\right)} .
$$

Transform the coordinate again and we get

$$
L^{2} \int d \lambda_{1} d \lambda_{2} \frac{\sin ^{2}\left(\pi L\left(\lambda_{1}-\lambda_{2}\right) \rho\left(\left(\lambda_{1}+\lambda_{2}\right) / 2\right)\right)}{\left(\pi L\left(\lambda_{i}-\lambda_{j}\right)\right)^{2}} e^{i\left(\lambda_{1}-\lambda_{2}\right) t} e^{-\beta\left(\lambda_{1}+\lambda_{2}\right)}=L^{2} \int d u_{1} d u_{2} \frac{\sin ^{2}\left(\pi L u_{1} \rho\left(u_{2}\right)\right)}{\left(\pi L u_{1}\right)^{2}} e^{i u_{1} t} e^{-2 \beta u_{2}} .
$$

The small interval contribution will again give 


$$
L^{2} \int_{-\Omega_{0} / 2}^{\Omega_{0} / 2} d u_{1} \frac{\sin ^{2}\left(\pi L u_{1} \rho\left(u_{2}\right)\right)}{\left(\pi L u_{1}\right)^{2}} e^{i u_{1} t} e^{-2 \beta u_{2}} \sim e^{-2 \beta u_{2}} \max \left(L \rho\left(u_{2}\right)-\frac{t}{2 \pi}, 0\right) .
$$

We cannot find an analytic formula for a general $\beta$ if we wish to compute this integral over $u_{2}$. However, one can expand it over a small $\beta$. We have

$$
L^{2} \int d u_{1} d u_{2} \frac{\sin ^{2}\left(\pi L u_{1} \rho\left(u_{2}\right)\right)}{\left(\pi L u_{1}\right)^{2}} e^{i u_{1} t} e^{-2 \beta u_{2}}= \begin{cases}\frac{2}{\pi} L \operatorname{arccsc}\left(\frac{2 L}{\sqrt{4 L^{2}-t^{2}}}\right)-\frac{t}{2 \pi L} \sqrt{4 L^{2}-t^{2}} & \text { for } t<2 L \\ +\frac{\beta^{2}\left(-10 L^{2} t \sqrt{4 L^{2}-t^{2}}+t^{3} \sqrt{4 L^{2}-t^{2}}+24 L^{4} \operatorname{arccsc}\left(\frac{2 L}{\sqrt{4 L^{2}-t^{2}}}\right)\right.}{6 \pi L^{3}}+\mathcal{O}\left(\beta^{4}\right) & \text { for } t>2 L .\end{cases}
$$

\section{GOE/GSE SPECTRAL FORM FACTOR}

\section{A. Random matrix theory review}

GOEs and GSEs describe physical systems with discrete antiunitary symmetries. Here, we will briefly review the mathematical construction. We define the joint distribution of eigenvalues for GOEs and GSEs as

$$
P\left(\lambda_{1}, \ldots, \lambda_{L}\right) \sim e^{-\tilde{\beta} \frac{L}{4} \sum_{i} \lambda_{i}^{2}} \prod_{i<j}\left(\lambda_{i}-\lambda_{j}\right)^{\tilde{\beta}},
$$

where $\tilde{\beta}=1,4$ for a GOE or a GSE. Here, our convention is $L \times L$ for a GOE and $2 L \times 2 L$ for a GSE (where the later only has $L$ independent eigenvalues because it has a natural degeneracy of two by construction). Again, we define the $n$-point correlation function as

$$
\rho^{(n)}\left(\lambda_{1}, \ldots, \lambda_{n}\right)=\int d \lambda_{n+1} \ldots d \lambda_{L} P\left(\lambda_{1}, \ldots, \lambda_{L}\right) .
$$

To go further, we need some quaternion matrix theories. A quaternion is generated by four units $e_{1,2,3}$,

$$
q=q^{(0)}+q^{(1)} e_{1}+q^{(2)} e_{2}+q^{(3)} e_{3} .
$$

The units are defined to satisfy the following multiplication laws

$$
\begin{aligned}
1 \times e_{j} & =e_{j} \times 1=e_{j} \\
e_{1}^{2} & =e_{2}^{2}=e_{3}^{2}=e_{1} e_{2} e_{3}=-1 .
\end{aligned}
$$

These units have matrix representations:

$$
\begin{aligned}
1 \rightarrow\left(\begin{array}{cc}
1 & 0 \\
0 & 1
\end{array}\right) & e_{1} \rightarrow\left(\begin{array}{cc}
i & 0 \\
0 & -i
\end{array}\right) \\
e_{2} \rightarrow\left(\begin{array}{cc}
0 & 1 \\
-1 & 0
\end{array}\right) & e_{3} \rightarrow\left(\begin{array}{ll}
0 & i \\
i & 0
\end{array}\right) .
\end{aligned}
$$

The determinant of a $n \times n$ quaternion matrix $Q=\left(Q_{j k}\right)$ is defined as

$$
\operatorname{det} Q=\sum_{\sigma}(-1)^{n-\ell(\sigma, Q)} \prod_{t=1}^{\ell(\sigma, Q)} \operatorname{cyc}_{t}(\sigma, Q)
$$

where $\sigma$ is any possible permutations from 1 to $n$. For corresponding permutations, we could find all $\ell$ closed cycles for those permutations. For instance, for a cycle $t$ like

$$
t: a \rightarrow b \rightarrow c \rightarrow \cdots \rightarrow d \rightarrow a,
$$

the corresponding contribution in the product is

$$
\operatorname{cyc}_{t}(\sigma, Q)=\left(q_{a b} q_{b c} \cdots q_{c d} q_{d a}\right)^{(0)},
$$

where the upper index (0) means the scalar part, or equivalently

$$
\left(q_{a b} q_{b c} \cdots q_{c d} q_{d a}\right)^{(0)}=\frac{1}{2} \operatorname{Tr}\left(q_{a b} q_{b c} \cdots q_{c d} q_{d a}\right) .
$$

There are some useful theorems to compute the quaternion determinant. For instance, if we use the matrix representation of the quaternion by replacing $e_{j}$ with $2 \times 2$ matrices, we can define a $2 n \times 2 n$ complex matrix $C(Q)$ for a $n \times n$ quaternion matrix $Q$. Now, define $Z=C\left(e_{2} I_{n}\right)$, where $I_{n}$ is the $n \times n$ unit matrix. Then, if $Q$ is a self-dual matrix, namely, each cycle from the product in the definition of the determinant is reversible (dual to each other), then one can show that

$$
\operatorname{det} Q=\operatorname{Pf}(Z C(Q))=\operatorname{det}^{1 / 2}(C(Q)) .
$$

With these definitions, we could define the quaternion kernels for a GOE and a GSE. In a GOE and a GSE, the sine kernel $K$ is replaced by a quaternion, which could be represented as a $2 \times 2$ matrix. In fact, we define the following function: 


$$
\begin{array}{ccc}
\hat{s}(r) & =\frac{\sin (r)}{r} & \epsilon(r)=\frac{1}{2} \operatorname{sign}(r) \\
\mathbf{D} \hat{s}(r)=\partial_{r} \hat{s}(r) & \mathbf{I} \hat{s}(r)=\int_{0}^{r} \hat{s}(t) d t .
\end{array}
$$

Thus, the quaternion kernel for a GOE is

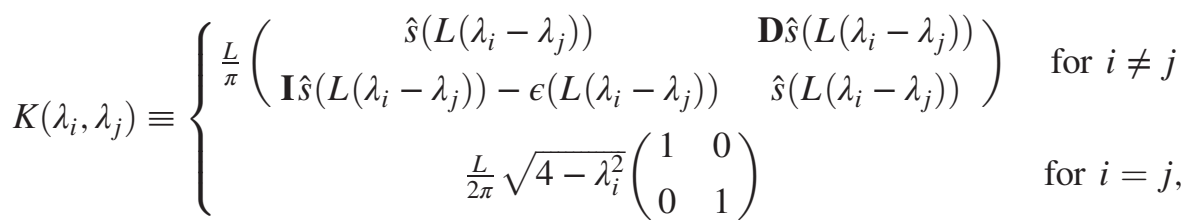

while for a GSE it is

$$
K\left(\lambda_{i}, \lambda_{j}\right) \equiv\left\{\begin{array}{cc}
\frac{L}{\pi}\left(\begin{array}{cc}
\hat{s}\left(2 L\left(\lambda_{i}-\lambda_{j}\right)\right) & \mathbf{D} \hat{s}\left(2 L\left(\lambda_{i}-\lambda_{j}\right)\right) \\
\mathbf{I} \hat{s}\left(2 L\left(\lambda_{i}-\lambda_{j}\right)\right) & \hat{s}\left(2 L\left(\lambda_{i}-\lambda_{j}\right)\right)
\end{array}\right) & \text { for } i \neq j \\
\frac{L}{2 \pi} \sqrt{4-\lambda_{i}^{2}}\left(\begin{array}{cc}
1 & 0 \\
0 & 1
\end{array}\right) & \text { for } i=j
\end{array}\right.
$$

The structure of a GOE and a GSE is not called simply determining the ordinary meaning of a determinant of some two-point functions. It is not called the determinantal point process in random matrix theory literature, but it is called the Pfaffian point process. For our practical motivation, we may define the joint eigenvalue distribution as some linear combination of the cluster function $T$,

$\rho^{(n)}\left(\lambda_{1}, \ldots, \lambda_{n}\right)=\frac{(L-n) !}{L !} \sum_{m \geq 1}^{\cup_{i=1}^{m} S_{i}=\{1, \ldots, n\}}(-1)^{n-m} T_{S_{1}} \ldots T_{S_{m}}$

where $T_{S}=T_{l}\left(x_{i_{1}}, x_{i_{2}}, \cdots, x_{i_{l}}\right)$ and $S=\left\{i_{1}, i_{2}, \cdots, i_{l}\right\}$, and the sum is over all possible decompositions of $\{1, \ldots, n\}$ (৬ means disjoint union). For instance,

$\rho^{(2)}\left(\lambda_{1}, \lambda_{2}\right)=\frac{1}{L(L-1)}\left(T_{1}\left(\lambda_{1}\right) T_{1}\left(\lambda_{2}\right)-T_{2}\left(\lambda_{1}, \lambda_{2}\right)\right)$.

The cluster function could be computed by the quaternion kernel as

$$
\begin{aligned}
T_{n}\left(\lambda_{1}, \ldots, \lambda_{n}\right)= & \frac{1}{2 n} \sum_{\sigma} \operatorname{Tr}\left(K\left(\lambda_{\sigma_{1}}, \lambda_{\sigma_{2}}\right)\right. \\
& \left.\times K\left(\lambda_{\sigma_{2}}, \lambda_{\sigma_{3}}\right) \ldots K\left(\lambda_{\sigma_{n}}, \lambda_{\sigma_{1}}\right)\right),
\end{aligned}
$$

where the sum is taken over all permutations $\sigma$ of $\{1, \ldots, n\}$. Thus, from these computations, we could, in principle, reduce the correlation functions into cluster functions, and then the products of trace of kernels, which are essentially computable. There are some of the simplest examples for those formulas, for instance,

$$
\begin{aligned}
\rho^{(1)}\left(\lambda_{1}\right)= & \frac{1}{L} \times \frac{1}{2} \operatorname{Tr}\left(K\left(\lambda_{1}, \lambda_{1}\right)\right) \\
\rho^{(2)}\left(\lambda_{1}, \lambda_{2}\right)= & \frac{1}{L(L-1)} \times\left(\frac{1}{4} \operatorname{Tr}\left(K\left(\lambda_{1}, \lambda_{1}\right)\right) \operatorname{Tr}\left(K\left(\lambda_{2}, \lambda_{2}\right)\right)\right. \\
& \left.-\frac{1}{2} \operatorname{Tr}\left(K\left(\lambda_{1}, \lambda_{2}\right) K\left(\lambda_{2}, \lambda_{1}\right)\right)\right) .
\end{aligned}
$$

With this knowledge, we could start to compute spectral form factors.

\section{B. Form factor computation with box approximation}

\section{Theorems}

It is straightforward to generalize our previous formula of convolution kernels to the quaternion matrix theory. We have

Theorem 3.1 (Convolution formula for GOE): We have

$$
\begin{aligned}
& \int \prod_{i=1}^{m} d \lambda_{i} K\left(\lambda_{1}, \lambda_{2}\right) K\left(\lambda_{2}, \lambda_{3}\right) \ldots K\left(\lambda_{m-1}, \lambda_{m}\right) \\
& \times K\left(\lambda_{m}, \lambda_{1}\right) e^{i \sum_{i=1}^{m} k_{i} \lambda_{i}} \\
& =\operatorname{Lr}_{3}\left(\sum_{i=1}^{m} k_{i}\right) \int d k G(k) G\left(k+\frac{k_{1}}{2 L}\right) \\
& \quad \times G\left(k+\frac{k_{2}}{2 L}\right) \ldots G\left(k+\frac{k_{m-1}}{2 L}\right),
\end{aligned}
$$


where

$$
G(k)=\left(\begin{array}{cc}
g(k) & (-2 \pi i k) g(k) \\
\frac{g(k)-1}{-2 \pi i k} & g(k)
\end{array}\right),
$$

and

Theorem 3.2 (Convolution formula for GSE): We have

$$
\begin{aligned}
& \int \prod_{i=1}^{m} d \lambda_{i} K\left(\lambda_{1}, \lambda_{2}\right) K\left(\lambda_{2}, \lambda_{3}\right) \ldots K\left(\lambda_{m-1}, \lambda_{m}\right) \\
& \quad \times K\left(\lambda_{m}, \lambda_{1}\right) e^{i \sum_{i=1}^{m} k_{i} \lambda_{i}} \\
& =\operatorname{Lr}_{3}\left(\sum_{i=1}^{m} k_{i}\right) \int d k H(k) H\left(k+\frac{k_{1}}{2 L}\right) \\
& \quad \times H\left(k+\frac{k_{2}}{2 L}\right) \ldots H\left(k+\frac{k_{m-1}}{2 L}\right),
\end{aligned}
$$

where

$$
H(k)=\frac{1}{2} g\left(\frac{k}{2}\right)\left(\begin{array}{cc}
1 & -\pi i k \\
\frac{1}{-\pi i k} & 1
\end{array}\right)
$$

The original infinite $L$ versions of these formulas are hidden in Eqs. (6.4.21) and (7.2.10) in [7].

\section{Two-point form factor}

Based on our GUE knowledge, we will briefly describe how to compute form factors.

We start by computing $\mathcal{R}_{2}$ at infinite temperature for a GOE,

$$
\begin{aligned}
\mathcal{R}_{2}(t)= & L+\int d \lambda_{1} d \lambda_{2}\left(\frac{1}{4} \operatorname{Tr}\left(K\left(\lambda_{1}, \lambda_{1}\right)\right)\right. \\
& \left.\times \operatorname{Tr}\left(K\left(\lambda_{2}, \lambda_{2}\right)\right)-\frac{1}{2} \operatorname{Tr}\left(K\left(\lambda_{1}, \lambda_{2}\right) K\left(\lambda_{2}, \lambda_{1}\right)\right)\right) e^{i\left(\lambda_{1}-\lambda_{2}\right) t}
\end{aligned}
$$

Evaluating the first term in the integral, we find

$$
\begin{aligned}
& \int d \lambda_{1} d \lambda_{2}\left(\frac{1}{4} \operatorname{Tr}\left(K\left(\lambda_{1}, \lambda_{1}\right)\right) \operatorname{Tr}\left(K\left(\lambda_{2}, \lambda_{2}\right)\right)\right) e^{i\left(\lambda_{1}-\lambda_{2}\right) t} \\
& =L^{2} r_{1}^{2}(t) .
\end{aligned}
$$

The second term can be evaluated as

$$
\int d \lambda_{1} d \lambda_{2}\left(\frac{1}{2} \operatorname{Tr}\left(K\left(\lambda_{1}, \lambda_{2}\right) K\left(\lambda_{2}, \lambda_{1}\right)\right)\right) e^{i\left(\lambda_{1}-\lambda_{2}\right) t}=L r_{2}(t),
$$

where

$$
r_{2}(t)= \begin{cases}1-\frac{t}{L}+\frac{t}{2 L} \log \left(1+\frac{t}{L}\right) & t<2 L \\ -1+\frac{t}{2 L} \log \left(\frac{t+L}{t-L}\right) & t>2 L\end{cases}
$$

The final result is

$$
\mathcal{R}_{2}(t)=L+L^{2} r_{1}^{2}(t)-L r_{2}(t)
$$

In a GSE, the only difference between a GOE and a GSE is

$$
\begin{aligned}
& \int d \lambda_{1} d \lambda_{2}\left(\frac{1}{2} \operatorname{Tr}\left(K\left(\lambda_{1}, \lambda_{2}\right) K\left(\lambda_{2}, \lambda_{1}\right)\right)\right) e^{i\left(\lambda_{1}-\lambda_{2}\right) t} \\
& =L \begin{cases}1-\frac{1}{2} \cdot \frac{t}{2 L}+\frac{1}{4} \cdot \frac{t}{2 L} \cdot \log \left|1-\frac{t}{2 L}\right| & t<4 L \\
0 & t>4 L .\end{cases}
\end{aligned}
$$

This integration is, in fact, divergent between $0<t \leq 2 L$. It is because of a pole $1 / k$ in the expression of $H(k)$. However, that is an artifact of the Fourier transformation of the integral of the sine kernel $\operatorname{sinc}(x)$. Besides the methods of explicitly computing the Fourier transformation, we could also understand the time before $2 L$ as a continuation. As a result, there is a pole at $t=2 L$.

So as a conclusion, in a GSE we have to replace the result of $r_{2}$ by

$$
r_{2}= \begin{cases}1-\frac{t}{4 L}+\frac{t}{8 L} \log \left|1-\frac{t}{2 L}\right| & t<4 L \\ 0 & t>4 L .\end{cases}
$$

\section{Four-point form factor}

In this part, we need to compute $\mathcal{R}_{4}$ in a GOE, which is

$$
\mathcal{R}_{4}=\sum_{a, b, c, d=1}^{L} \int D \lambda e^{i\left(\lambda_{a}+\lambda_{b}-\lambda_{c}-\lambda_{d}\right) t}
$$

Take a look at the classifications of combinations in $\mathcal{R}_{4}$, which are:

(i) $a=b=c=d=e=f$ : Contribute $L$.

(ii) $a=b$ : Contribute $L(L-1)(L-2) \int D \lambda e^{i\left(2 \lambda_{1}-\lambda_{2}-\lambda_{3}\right) t}$.

(iii) $c=d$ : Contribute $L(L-1)(L-2) \int D \lambda e^{i\left(\lambda_{1}+\lambda_{2}-2 \lambda_{3}\right) t}$.

(iv) $a=c$ or $a=d$ or $b=c$ or $b=d$ : Contribute $4 L(L-1)(L-2) \int D \lambda e^{i\left(\lambda_{1}-\lambda_{2}\right) t}$.

(v) $b=c=d \quad$ or $\quad a=c=d \quad$ or $\quad a=b=d \quad$ or $a=b=c$ : Contribute $4 L(L-1) \int D \lambda e^{i\left(\lambda_{1}-\lambda_{2}\right) t}$.

(vi) $a=b \quad$ and $\quad c=d$ : Contribute $L(L-1)$ $\int D \lambda e^{i\left(2 \lambda_{1}-2 \lambda_{2}\right) t}$.

(vii) $a=c$ and $b=d$, or $a=d$ and $b=c$ : Contribute $2 L(L-1)$.

(viii) All inequal indexes: $L(L-1)(L-2)(L-3)$ $\int D \lambda e^{i\left(\lambda_{1}+\lambda_{2}-\lambda_{3}-\lambda_{4}\right) t}$.

Adding the total prefactors will give $L^{4}$. Add them together and we get 


$$
\begin{aligned}
\mathcal{R}_{4}= & L(L-1)(L-2)(L-3) \int D \lambda e^{i\left(\lambda_{1}+\lambda_{2}-\lambda_{3}-\lambda_{4}\right) t}+2 L(L-1)(L-2) \operatorname{Re} \int D \lambda e^{i\left(2 \lambda_{1}-\lambda_{2}-\lambda_{3}\right) t} \\
& +L(L-1) \int D \lambda e^{i\left(2 \lambda_{1}-2 \lambda_{2}\right) t}+4 L(L-1)^{2} \int D \lambda e^{i\left(\lambda_{1}-\lambda_{2}\right) t}+2 L^{2}-L \\
= & L(L-1)(L-2)(L-3) \int D \lambda e^{i\left(\lambda_{1}+\lambda_{2}-\lambda_{3}-\lambda_{4}\right) t}+2 L(L-1)(L-2) \operatorname{Re} \int D \lambda e^{i\left(2 \lambda_{1}-\lambda_{2}-\lambda_{3}\right) t} \\
& +L^{2} r_{1}^{2}(2 t)-L r_{2}(2 t)+4(L-1)\left(L^{2} r_{1}^{2}(t)-L r_{2}(t)\right)+2 L^{2}-L .
\end{aligned}
$$

We have already obtained what the last three terms are. Now we only need to consider the first two terms.

The first term.-The first term is an actual four-point function,

$$
L(L-1)(L-2)(L-3) \int D \lambda e^{i\left(\lambda_{1}+\lambda_{2}-\lambda_{3}-\lambda_{4}\right) t}
$$

In order to compute it, we will use the following decomposition from the correlation function to cluster function

$$
\begin{aligned}
L(L-1)(L-2)(L-3) \rho^{(4)}\left(\lambda_{1}, \lambda_{2}, \lambda_{3}, \lambda_{4}\right)= & -T_{4}\left(\lambda_{1}, \lambda_{2}, \lambda_{3}, \lambda_{4}\right)+T_{3}\left(\lambda_{2}, \lambda_{3}, \lambda_{4}\right) T_{1}\left(\lambda_{1}\right)+T_{3}\left(\lambda_{1}, \lambda_{3}, \lambda_{4}\right) T_{1}\left(\lambda_{2}\right) \\
& +T_{3}\left(\lambda_{1}, \lambda_{2}, \lambda_{4}\right) T_{1}\left(\lambda_{3}\right)+T_{3}\left(\lambda_{1}, \lambda_{2}, \lambda_{3}\right) T_{1}\left(\lambda_{4}\right)+T_{2}\left(\lambda_{1}, \lambda_{2}\right) T_{2}\left(\lambda_{3}, \lambda_{4}\right) \\
& +T_{2}\left(\lambda_{1}, \lambda_{3}\right) T_{2}\left(\lambda_{2}, \lambda_{4}\right)+T_{2}\left(\lambda_{1}, \lambda_{4}\right) T_{2}\left(\lambda_{2}, \lambda_{3}\right)-T_{1}\left(\lambda_{1}\right) T_{1}\left(\lambda_{2}\right) T_{2}\left(\lambda_{3}, \lambda_{4}\right) \\
& -T_{1}\left(\lambda_{1}\right) T_{1}\left(\lambda_{3}\right) T_{2}\left(\lambda_{2}, \lambda_{4}\right)-T_{1}\left(\lambda_{1}\right) T_{1}\left(\lambda_{4}\right) T_{2}\left(\lambda_{2}, \lambda_{3}\right)-T_{1}\left(\lambda_{2}\right) T_{1}\left(\lambda_{3}\right) T_{2}\left(\lambda_{1}, \lambda_{4}\right) \\
& -T_{1}\left(\lambda_{2}\right) T_{1}\left(\lambda_{4}\right) T_{2}\left(\lambda_{1}, \lambda_{3}\right)-T_{1}\left(\lambda_{3}\right) T_{1}\left(\lambda_{4}\right) T_{2}\left(\lambda_{1}, \lambda_{2}\right) \\
& +T_{1}\left(\lambda_{1}\right) T_{1}\left(\lambda_{2}\right) T_{1}\left(\lambda_{3}\right) T_{1}\left(\lambda_{4}\right)
\end{aligned}
$$

From the previous discussions, we have

$$
\begin{aligned}
T_{1}\left(\lambda_{a}\right)= & \frac{1}{2} \operatorname{Tr}\left(K\left(\lambda_{a}, \lambda_{a}\right)\right) \\
T_{2}\left(\lambda_{a}, \lambda_{b}\right)= & \frac{1}{2} \operatorname{Tr}\left(K\left(\lambda_{a}, \lambda_{b}\right) K\left(\lambda_{b}, \lambda_{a}\right)\right) \\
T_{3}\left(\lambda_{a}, \lambda_{b}, \lambda_{c}\right)= & \frac{1}{2} \operatorname{Tr}\left(K\left(\lambda_{a}, \lambda_{b}\right) K\left(\lambda_{b}, \lambda_{c}\right) K\left(\lambda_{c}, \lambda_{a}\right)\right)+\frac{1}{2} \operatorname{Tr}\left(K\left(\lambda_{a}, \lambda_{c}\right) K\left(\lambda_{c}, \lambda_{b}\right) K\left(\lambda_{b}, \lambda_{a}\right)\right) \\
T_{4}\left(\lambda_{a}, \lambda_{b}, \lambda_{c}, \lambda_{d}\right)= & \frac{1}{2} \operatorname{Tr}\left(K\left(\lambda_{a}, \lambda_{b}\right) K\left(\lambda_{b}, \lambda_{c}\right) K\left(\lambda_{c}, \lambda_{d}\right) K\left(\lambda_{d}, \lambda_{a}\right)\right)+\frac{1}{2} \operatorname{Tr}\left(K\left(\lambda_{a}, \lambda_{b}\right) K\left(\lambda_{b}, \lambda_{d}\right) K\left(\lambda_{d}, \lambda_{c}\right) K\left(\lambda_{c}, \lambda_{a}\right)\right) \\
& +\frac{1}{2} \operatorname{Tr}\left(K\left(\lambda_{a}, \lambda_{c}\right) K\left(\lambda_{c}, \lambda_{b}\right) K\left(\lambda_{b}, \lambda_{d}\right) K\left(\lambda_{d}, \lambda_{a}\right)\right)+\frac{1}{2} \operatorname{Tr}\left(K\left(\lambda_{a}, \lambda_{c}\right) K\left(\lambda_{c}, \lambda_{d}\right) K\left(\lambda_{d}, \lambda_{b}\right) K\left(\lambda_{b}, \lambda_{a}\right)\right) \\
& +\frac{1}{2} \operatorname{Tr}\left(K\left(\lambda_{a}, \lambda_{d}\right) K\left(\lambda_{d}, \lambda_{b}\right) K\left(\lambda_{b}, \lambda_{c}\right) K\left(\lambda_{c}, \lambda_{a}\right)\right)+\frac{1}{2} \operatorname{Tr}\left(K\left(\lambda_{a}, \lambda_{d}\right) K\left(\lambda_{d}, \lambda_{c}\right) K\left(\lambda_{c}, \lambda_{b}\right) K\left(\lambda_{b}, \lambda_{a}\right)\right),
\end{aligned}
$$

where we have already used the property of cyclic invariance for the trace operator. We can separately discuss these terms as the following:

(i) 4-type: In this case, we only have the $T_{4}$. Also in this case, all six terms in the expansion give the same answer, which is

$$
-\int d \lambda_{1} d \lambda_{2} d \lambda_{3} d \lambda_{4} T_{4}\left(\lambda_{1}, \lambda_{2}, \lambda_{3}, \lambda_{4}\right) e^{i\left(\lambda_{1}+\lambda_{2}-\lambda_{3}-\lambda_{4}\right) t}=-6 L r_{4}(t)
$$


where

$$
r_{4}(t)= \begin{cases}1-\frac{7 t}{4 L}+\frac{5 t}{4 L} \log \left(1+\frac{t}{L}\right) & 0<t<L \\ -\frac{3}{2}+\frac{3 t}{4 L}+\frac{5 t}{4 L} \log \left(\frac{1}{2}\left(1+\frac{3 L}{2 t-L}\right)\right) & L<t<2 L \\ 0 & t>2 L\end{cases}
$$

(ii) 1-1-1-1-type: In this case, we only have $T_{1}$. Thus, we have

$$
\int d \lambda_{1} d \lambda_{2} d \lambda_{3} d \lambda_{4} T_{1}\left(\lambda_{1}\right) T_{1}\left(\lambda_{2}\right) T_{1}\left(\lambda_{3}\right) T_{1}\left(\lambda_{4}\right) e^{i\left(\lambda_{1}+\lambda_{2}-\lambda_{3}-\lambda_{4}\right) t}=L^{4} r_{1}^{4}(t)
$$

(iii) 1-1-2-type: In this case, we have both $T_{1}$ and $T_{2}$. Thus, we have

$$
\begin{aligned}
& -\int d \lambda_{1} d \lambda_{2} d \lambda_{3} d \lambda_{4} T_{1}\left(\lambda_{1}\right) T_{1}\left(\lambda_{2}\right) T_{2}\left(\lambda_{3}, \lambda_{4}\right) e^{i\left(\lambda_{1}+\lambda_{2}-\lambda_{3}-\lambda_{4}\right) t}-\int d \lambda_{1} d \lambda_{2} d \lambda_{3} d \lambda_{4} T_{1}\left(\lambda_{1}\right) T_{1}\left(\lambda_{3}\right) T_{2}\left(\lambda_{2}, \lambda_{4}\right) e^{i\left(\lambda_{1}+\lambda_{2}-\lambda_{3}-\lambda_{4}\right) t} \\
& -\int d \lambda_{1} d \lambda_{2} d \lambda_{3} d \lambda_{4} T_{1}\left(\lambda_{1}\right) T_{1}\left(\lambda_{4}\right) T_{2}\left(\lambda_{2}, \lambda_{3}\right) e^{i\left(\lambda_{1}+\lambda_{2}-\lambda_{3}-\lambda_{4}\right) t}-\int d \lambda_{1} d \lambda_{2} d \lambda_{3} d \lambda_{4} T_{1}\left(\lambda_{2}\right) T_{1}\left(\lambda_{3}\right) T_{2}\left(\lambda_{1}, \lambda_{4}\right) e^{i\left(\lambda_{1}+\lambda_{2}-\lambda_{3}-\lambda_{4}\right) t} \\
& -\int d \lambda_{1} d \lambda_{2} d \lambda_{3} d \lambda_{4} T_{1}\left(\lambda_{2}\right) T_{1}\left(\lambda_{4}\right) T_{2}\left(\lambda_{1}, \lambda_{3}\right) e^{i\left(\lambda_{1}+\lambda_{2}-\lambda_{3}-\lambda_{4}\right) t}-\int d \lambda_{1} d \lambda_{2} d \lambda_{3} d \lambda_{4} T_{1}\left(\lambda_{3}\right) T_{1}\left(\lambda_{4}\right) T_{2}\left(\lambda_{1}, \lambda_{2}\right) e^{i\left(\lambda_{1}+\lambda_{2}-\lambda_{3}-\lambda_{4}\right) t} \\
& \quad=-2 L^{3} r_{1}^{2}(t) r_{3}(2 t) r_{2}(t)-4 L^{3} r_{1}^{2}(t) r_{2}(t) .
\end{aligned}
$$

(iv) 2-2-type: In this case, we only have $T_{2}$. Thus, we have

$$
\begin{aligned}
& +\int d \lambda_{1} d \lambda_{2} d \lambda_{3} d \lambda_{4} T_{2}\left(\lambda_{1}, \lambda_{2}\right) T_{2}\left(\lambda_{3}, \lambda_{4}\right) e^{i\left(\lambda_{1}+\lambda_{2}-\lambda_{3}-\lambda_{4}\right) t}+\int d \lambda_{1} d \lambda_{2} d \lambda_{3} d \lambda_{4} T_{2}\left(\lambda_{1}, \lambda_{3}\right) T_{2}\left(\lambda_{2}, \lambda_{4}\right) e^{i\left(\lambda_{1}+\lambda_{2}-\lambda_{3}-\lambda_{4}\right) t} \\
& +\int d \lambda_{1} d \lambda_{2} d \lambda_{3} d \lambda_{4} T_{2}\left(\lambda_{1}, \lambda_{4}\right) T_{2}\left(\lambda_{2}, \lambda_{3}\right) e^{i\left(\lambda_{1}+\lambda_{2}-\lambda_{3}-\lambda_{4}\right) t}=2 L^{2} r_{2}^{2}(t)+L^{2} r_{2}^{2}(t) r_{3}^{2}(2 t) .
\end{aligned}
$$

(v) 3-1-type: In this case, we have both $T_{3}$ and $T_{1}$. Thus, we have

$$
\begin{aligned}
& +\int d \lambda_{1} d \lambda_{2} d \lambda_{3} d \lambda_{4} T_{3}\left(\lambda_{2}, \lambda_{3}, \lambda_{4}\right) T_{1}\left(\lambda_{1}\right) e^{i\left(\lambda_{1}+\lambda_{2}-\lambda_{3}-\lambda_{4}\right) t}+\int d \lambda_{1} d \lambda_{2} d \lambda_{3} d \lambda_{4} T_{3}\left(\lambda_{1}, \lambda_{3}, \lambda_{4}\right) T_{1}\left(\lambda_{2}\right) e^{i\left(\lambda_{1}+\lambda_{2}-\lambda_{3}-\lambda_{4}\right) t} \\
& +\int d \lambda_{1} d \lambda_{2} d \lambda_{3} d \lambda_{4} T_{3}\left(\lambda_{1}, \lambda_{2}, \lambda_{4}\right) T_{1}\left(\lambda_{3}\right) e^{i\left(\lambda_{1}+\lambda_{2}-\lambda_{3}-\lambda_{4}\right) t}+\int d \lambda_{1} d \lambda_{2} d \lambda_{3} d \lambda_{4} T_{3}\left(\lambda_{1}, \lambda_{2}, \lambda_{3}\right) T_{1}\left(\lambda_{4}\right) e^{i\left(\lambda_{1}+\lambda_{2}-\lambda_{3}-\lambda_{4}\right) t} \\
& \quad=6 L^{2} r_{1}(t) r_{3,1}(t) r_{3}(t)+2 L^{2} r_{1}(t) r_{3,2}(t) r_{3}(t),
\end{aligned}
$$

where

$$
r_{3,1}(t)= \begin{cases}1-\frac{2 t}{L}+\frac{3 t}{2 L} \log \left(1+\frac{t}{L}\right) & 0<t<L \\ -2+\frac{t}{L}+\frac{3 t}{2 L} \log \left(\frac{1}{2}\left(1+\frac{3 L}{2 t-L}\right)\right) & L<t<2 L \\ 0 & t>2 L\end{cases}
$$

and

$$
r_{3,2}(t)=r_{2}(t)
$$


We have the total expression as

$$
\begin{aligned}
L(L-1)(L-2)(L-3) \int D \lambda e^{i\left(\lambda_{1}+\lambda_{2}-\lambda_{3}-\lambda_{4}\right) t}= & L^{4} r_{1}^{4}(t)-2 L^{3} r_{1}^{2}(t) r_{3}(2 t) r_{2}(t)-4 L^{3} r_{1}^{2}(t) r_{2}(t)+2 L^{2} r_{2}^{2}(t) \\
& +L^{2} r_{2}^{2}(t) r_{3}^{2}(2 t)+6 L^{2} r_{1}(t) r_{3,1}(t) r_{3}(t)+2 L^{2} r_{1}(t) r_{3,2}(t) r_{3}(t)-6 L r_{4}(t)
\end{aligned}
$$

The second term.- In this part, we will evaluate the second term

$$
2 L(L-1)(L-2) \operatorname{Re} \int D \lambda e^{i\left(2 \lambda_{1}-\lambda_{2}-\lambda_{3}\right) t} .
$$

Let us first consider it without a factor of 2 ,

$$
L(L-1)(L-2) \operatorname{Re} \int D \lambda e^{i\left(2 \lambda_{1}-\lambda_{2}-\lambda_{3}\right) t} .
$$

Do the same cluster decomposition,

$$
\begin{aligned}
L(L-1)(L-2) \rho^{(3)}\left(\lambda_{1}, \lambda_{2}, \lambda_{3}\right)= & T_{3}\left(\lambda_{1}, \lambda_{2}, \lambda_{3}\right)-T_{2}\left(\lambda_{2}, \lambda_{3}\right) T_{1}\left(\lambda_{1}\right)-T_{2}\left(\lambda_{1}, \lambda_{3}\right) T_{1}\left(\lambda_{2}\right)-T_{2}\left(\lambda_{1}, \lambda_{2}\right) T_{1}\left(\lambda_{3}\right) \\
& +T_{1}\left(\lambda_{1}\right) T_{1}\left(\lambda_{2}\right) T_{1}\left(\lambda_{3}\right) .
\end{aligned}
$$

Then, we obtain:

(i) 3-type: In this case, we have

$$
\operatorname{Re} \int d \lambda_{1} d \lambda_{2} d \lambda_{3} T_{3}\left(\lambda_{1}, \lambda_{2}, \lambda_{3}\right) e^{i\left(2 \lambda_{1}-\lambda_{2}-\lambda_{3}\right) t}=2 L r_{3,3}(t)
$$

where

$$
r_{3,3}(t)= \begin{cases}1-\frac{3 t}{L}+\frac{t}{L} \log \left(1+\frac{t}{L}\right)+\frac{5 t}{4 L} \log \left(1+\frac{2 t}{L}\right) & 0<t<\frac{2}{3} L \\ -2+\frac{3 t}{2 L}+\frac{t}{L} \log \left(\frac{L+t}{3 t-L}\right)+\frac{5 t}{4 L} \log \left(\frac{L+2 t}{3 t-L}\right) & \frac{2}{3} L<t<L \\ -1+\frac{t}{2 L}+\frac{5 t}{4 L} \log \left(\frac{L+2 t}{3 t-L}\right) & L<t<2 L \\ 0 & t>2 L\end{cases}
$$

(ii) 1-1-1-type: In this case, we have

$$
\operatorname{Re} \int d \lambda_{1} d \lambda_{2} d \lambda_{3} T_{1}\left(\lambda_{1}\right) T_{1}\left(\lambda_{2}\right) T_{1}\left(\lambda_{3}\right) e^{i\left(2 \lambda_{1}-\lambda_{2}-\lambda_{3}\right) t}=L^{3} r_{1}(2 t) r_{1}^{2}(t)
$$

(iii) 2-1-type: In this case, we have

$$
\begin{aligned}
& -\operatorname{Re} \int d \lambda_{1} d \lambda_{2} d \lambda_{3} T_{2}\left(\lambda_{2}, \lambda_{3}\right) T_{1}\left(\lambda_{1}\right) e^{i\left(2 \lambda_{1}-\lambda_{2}-\lambda_{3}\right) t}-\operatorname{Re} \int d \lambda_{1} d \lambda_{2} d \lambda_{3} T_{2}\left(\lambda_{1}, \lambda_{3}\right) T_{1}\left(\lambda_{2}\right) e^{i\left(2 \lambda_{1}-\lambda_{2}-\lambda_{3}\right) t} \\
& -\operatorname{Re} \int d \lambda_{1} d \lambda_{2} d \lambda_{3} T_{2}\left(\lambda_{1}, \lambda_{2}\right) T_{1}\left(\lambda_{3}\right) e^{i\left(2 \lambda_{1}-\lambda_{2}-\lambda_{3}\right) t}=-L^{2} r_{1}(2 t) r_{3}(2 t) r_{2}(t)-2 L^{2} r_{1}(t) r_{3}(t) r_{2}(2 t) .
\end{aligned}
$$


Finally, we can collect all of those terms together, and then we get

$2 L(L-1)(L-2) \operatorname{Re} \int D \lambda e^{i\left(2 \lambda_{1}-\lambda_{2}-\lambda_{3}\right) t}=2 L^{3} r_{1}(2 t) r_{1}^{2}(t)-2 L^{2} r_{1}(2 t) r_{3}(2 t) r_{2}(t)-4 L^{2} r_{1}(t) r_{3}(t) r_{2}(2 t)+4 L r_{3,3}(t)$.

Final expression and summary.-From those calculations, we could obtain the final expression for $\mathcal{R}_{4}$, which is

$$
\begin{aligned}
\mathcal{R}_{4}= & +L^{4} r_{1}^{4}(t)-2 L^{3} r_{1}^{2}(t) r_{2}(t) r_{3}(2 t)-4 L^{3} r_{1}^{2}(t) r_{2}(t)+2 L^{3} r_{1}(2 t) r_{1}^{2}(t)+4 L^{3} r_{1}^{2}(t)+2 L^{2} r_{2}^{2}(t)+L^{2} r_{2}^{2}(t) r_{3}^{2}(2 t) \\
& +6 L^{2} r_{1}(t) r_{3,1}(t) r_{3}(t)+2 L^{2} r_{1}(t) r_{3,2}(t) r_{3}(t)-2 L^{2} r_{1}(2 t) r_{3}(2 t) r_{2}(t)-4 L^{2} r_{1}(t) r_{3}(t) r_{2}(2 t)+L^{2} r_{1}^{2}(2 t)-4 L^{2} r_{1}^{2}(t) \\
& -4 L^{2} r_{2}(t)+2 L^{2}-6 L r_{4}(t)-L r_{2}(2 t)+4 L r_{3,3}(t)+4 L r_{2}(t)-L,
\end{aligned}
$$

where

$$
\begin{aligned}
& r_{2}(t)= \begin{cases}1-\frac{t}{L}+\frac{t}{2 L} \log \left(1+\frac{t}{L}\right) & t<2 L \\
-1+\frac{t}{2 L} \log \left(\frac{t+L}{t-L}\right) & t>2 L\end{cases} \\
& r_{3,1}(t)= \begin{cases}1-\frac{2 t}{L}+\frac{3 t}{2 L} \log \left(1+\frac{t}{L}\right) & 0<t<L \\
-2+\frac{t}{L}+\frac{3 t}{2 L} \log \left(\frac{1}{2}\left(1+\frac{3 L}{2 t-L}\right)\right) & L<t<2 L \\
0 & t>2 L\end{cases} \\
& r_{3,2}(t)=r_{2}(t) \\
& r_{3,3}(t)= \begin{cases}1-\frac{3 t}{L}+\frac{t}{L} \log \left(1+\frac{t}{L}\right)+\frac{5 t}{4 L} \log \left(1+\frac{2 t}{L}\right) & 0<t<\frac{2}{3} L \\
-2+\frac{3 t}{2 L}+\frac{t}{L} \log \left(\frac{L+t}{3 t-L}\right)+\frac{5 t}{4 L} \log \left(\frac{L+2 t}{3 t-L}\right) & \frac{2}{3} L<t<L \\
-1+\frac{t}{2 L}+\frac{5 t}{4 L} \log \left(\frac{L+2 t}{3 t-L}\right) & L<t<2 L \\
0 & t>2 L\end{cases} \\
& r_{4}(t)= \begin{cases}1-\frac{7 t}{4 L}+\frac{5 t}{4 L} \log \left(1+\frac{t}{L}\right) & 0<t<L \\
-\frac{3}{2}+\frac{3 t}{4 L}+\frac{5 t}{4 L} \log \left(\frac{1}{2}\left(1+\frac{3 L}{2 t-L}\right)\right) & L<t<2 L \\
0 & t>2 L .\end{cases}
\end{aligned}
$$

After dropping out the less-dominated terms, we could obtain

$$
\begin{aligned}
\mathcal{R}_{4} \sim & +L^{4} r_{1}^{4}(t)+2 L^{2} r_{2}^{2}(t)-4 L^{2} r_{2}(t)+2 L^{2}-6 L r_{4}(t) \\
& -L r_{2}(2 t)+4 L r_{3,3}(t)+4 L r_{2}(t)-L .
\end{aligned}
$$

GSE.-In a GSE, the computations are very similar, and we have to replace these block functions by

$$
\begin{gathered}
r_{4}(t)= \begin{cases}1-\frac{1}{2} \frac{t}{L}+\frac{3}{16} \frac{t}{L} \log \left|\frac{t}{L}-1\right| & t<2 L \\
0 & t>2 L\end{cases} \\
r_{3,1}=r_{4} \\
r_{3,2}=r_{2} \\
r_{3,3}= \begin{cases}1-\frac{3 t}{4 L}+\frac{t}{32 L} \log \left|\frac{2 t-2 L}{2 L-t}\right|+\frac{9 t}{32 L} \log \left|\frac{3}{2} \frac{t}{L}-1\right| & t<\frac{4}{3} L \\
0 & t>\frac{4}{3} L .50\end{cases}
\end{gathered}
$$

\section{Refined two-point form factor}

Now, we discuss the trick that is similar to our previous improvement. Let us start from a GOE. We will use the short distance refined kernel,

$$
\begin{array}{rlr}
\tilde{K}\left(\lambda_{i}, \lambda_{j}\right) \equiv & L \rho\left(\left(\lambda_{i}+\lambda_{j}\right) / 2\right) \\
& \times\left(\begin{array}{cc}
\hat{s}\left(\pi L \rho\left(\left(\lambda_{i}+\lambda_{j}\right) / 2\right)\left(\lambda_{i}-\lambda_{j}\right)\right) & \mathbf{D} \hat{s}\left(\pi L \rho\left(\left(\lambda_{i}+\lambda_{j}\right) / 2\right)\left(\lambda_{i}-\lambda_{j}\right)\right) \\
\mathbf{I} \hat{s}\left(\pi L \rho\left(\left(\lambda_{i}+\lambda_{j}\right) / 2\right)\left(\lambda_{i}-\lambda_{j}\right)\right)-\epsilon\left(\pi L \rho\left(\left(\lambda_{i}+\lambda_{j}\right) / 2\right)\left(\lambda_{i}-\lambda_{j}\right)\right) & \hat{s}\left(\pi L \rho\left(\left(\lambda_{i}+\lambda_{j}\right) / 2\right)\left(\lambda_{i}-\lambda_{j}\right)\right)
\end{array}\right) .
\end{array}
$$

We will try to use this formula to evaluate the two-point form factor, at a generic finite temperature, $\beta$ (while the refined infinite temperature form factor could be obtained by sending $\beta \rightarrow 0$ ). We have 


$$
\mathcal{R}_{2}(t, \beta)=L r_{1}(2 i \beta)+L^{2} r_{1}(t+i \beta) r_{1}(t-i \beta)-\frac{1}{2} \int d \lambda_{1} d \lambda_{2}\left(\operatorname{Tr}\left(K\left(\lambda_{1}, \lambda_{2}\right) K\left(\lambda_{2}, \lambda_{1}\right)\right)\right) e^{i\left(\lambda_{1}-\lambda_{2}\right) t} e^{-\beta\left(\lambda_{1}+\lambda_{2}\right)},
$$

while for the later integral, we expand it as

$$
\begin{aligned}
- & \frac{1}{2} \int d \lambda_{1} d \lambda_{2}\left(\operatorname{Tr}\left(K\left(\lambda_{1}, \lambda_{2}\right) K\left(\lambda_{2}, \lambda_{1}\right)\right)\right) e^{i\left(\lambda_{1}-\lambda_{2}\right) t} e^{-\beta\left(\lambda_{1}+\lambda_{2}\right)} \\
= & -L^{2} \int d \lambda_{1} d \lambda_{2} \frac{\sin ^{2}\left(\pi L \rho\left(\left(\lambda_{1}+\lambda_{2}\right) / 2\right)\left(\lambda_{1}-\lambda_{2}\right)\right)}{\left(\pi L\left(\lambda_{1}-\lambda_{2}\right)\right)^{2}} e^{i\left(\lambda_{1}-\lambda_{2}\right) t} e^{-\beta\left(\lambda_{1}+\lambda_{2}\right)} \\
& +L^{2} \int d \lambda_{1} d \lambda_{2} \rho^{2}\left(\frac{\lambda_{1}+\lambda_{2}}{2}\right)\left(\mathbf{D} \hat{s}\left(\pi L \rho\left(\frac{\lambda_{1}+\lambda_{2}}{2}\right)\left(\lambda_{1}-\lambda_{2}\right)\right) \mathbf{I} \hat{s}\left(\pi L \rho\left(\frac{\lambda_{1}+\lambda_{2}}{2}\right)\left(\lambda_{i}-\lambda_{j}\right)\right)\right) e^{i\left(\lambda_{1}-\lambda_{2}\right) t} e^{-\beta\left(\lambda_{1}+\lambda_{2}\right)} \\
& -L^{2} \int d \lambda_{1} d \lambda_{2} \rho^{2}\left(\frac{\lambda_{1}+\lambda_{2}}{2}\right)\left(\mathbf{D} \hat{s}\left(\pi L \rho\left(\frac{\lambda_{1}+\lambda_{2}}{2}\right)\left(\lambda_{1}-\lambda_{2}\right)\right) \epsilon\left(\pi L \rho\left(\frac{\lambda_{1}+\lambda_{2}}{2}\right)\left(\lambda_{i}-\lambda_{j}\right)\right)\right) e^{i\left(\lambda_{1}-\lambda_{2}\right) t} e^{-\beta\left(\lambda_{1}+\lambda_{2}\right)} .
\end{aligned}
$$

Again, changing the variable,

$$
u_{1}=\lambda_{1}-\lambda_{2} \quad u_{2}=\frac{\lambda_{1}+\lambda_{2}}{2}
$$

we simplify it as

$$
\begin{aligned}
-\frac{1}{2} \int d \lambda_{1} d \lambda_{2}\left(\operatorname{Tr}\left(K\left(\lambda_{1}, \lambda_{2}\right) K\left(\lambda_{2}, \lambda_{1}\right)\right)\right) e^{i\left(\lambda_{1}-\lambda_{2}\right) t} e^{-\beta\left(\lambda_{1}+\lambda_{2}\right)} & =-L^{2} \int d u_{1} d u_{2} \frac{\sin ^{2}\left(\pi L \rho\left(u_{2}\right) u_{1}\right)}{\left(\pi L u_{1}\right)^{2}} e^{i u_{1} t} e^{-2 \beta u_{2}} \\
& +L^{2} \int d u_{1} d u_{2} \rho^{2}\left(u_{2}\right)\left(\mathbf{D} \hat{s}\left(\pi L \rho\left(u_{2}\right) u_{1}\right) \mathbf{I} \hat{s}\left(\pi L \rho\left(u_{2}\right) u_{1}\right)\right) e^{i u_{1} t} e^{-2 \beta u_{2}} \\
& -L^{2} \int d u_{1} d u_{2} \rho^{2}\left(u_{2}\right)\left(\mathbf{D} \hat{s}\left(\pi L \rho\left(u_{2}\right) u_{1}\right) \epsilon\left(\pi L \rho\left(u_{2}\right) u_{1}\right)\right) e^{i u_{1} t} e^{-2 \beta u_{2}}
\end{aligned}
$$

We could first perform the integral over $u_{1}$, and the result is

$$
\begin{aligned}
& L e^{-2 \beta u_{2}} \rho\left(u_{2}\right) \begin{cases}1-\frac{t}{\pi \rho\left(u_{2}\right) L}+\frac{t}{2 \pi \rho\left(u_{2}\right) L} \log \left(1+\frac{t}{\pi \rho\left(u_{2}\right) L}\right) & t<2 \pi \rho\left(u_{2}\right) L \\
-1+\frac{t}{2 \pi \rho\left(u_{2}\right) L} \log \left(\frac{1+\frac{t}{\pi \rho\left(u_{2}\right) L}}{\frac{t}{\pi \rho\left(u_{2}\right) L}-1}\right) & t>2 \pi \rho\left(u_{2}\right) L\end{cases} \\
& =e^{-2 \beta u_{2}} \max \left(L \rho\left(u_{2}\right)-\frac{t}{\pi}+\frac{t}{2 \pi} \log \left(1+\frac{t}{\pi \rho\left(u_{2}\right) L}\right),-L \rho\left(u_{2}\right)+\frac{t}{2 \pi} \log \left(\frac{1+\frac{t}{\pi \rho\left(u_{2}\right) L}}{\frac{t}{\pi \rho\left(u_{2}\right) L}-1}\right)\right) .
\end{aligned}
$$

In the GSE case, we have the refined kernel,

$$
\tilde{K}\left(\lambda_{i}, \lambda_{j}\right) \equiv L \rho\left(\left(\lambda_{i}+\lambda_{j}\right) / 2\right)\left(\begin{array}{cc}
\hat{s}\left(2 \pi L \rho\left(\left(\lambda_{i}+\lambda_{j}\right) / 2\right)\left(\lambda_{i}-\lambda_{j}\right)\right) & \mathbf{D} \hat{s}\left(2 \pi L \rho\left(\left(\lambda_{i}+\lambda_{j}\right) / 2\right)\left(\lambda_{i}-\lambda_{j}\right)\right) \\
\mathbf{I} \hat{s}\left(2 \pi L \rho\left(\left(\lambda_{i}+\lambda_{j}\right) / 2\right)\left(\lambda_{i}-\lambda_{j}\right)\right) & \hat{s}\left(2 \pi L \rho\left(\left(\lambda_{i}+\lambda_{j}\right) / 2\right)\left(\lambda_{i}-\lambda_{j}\right)\right)
\end{array}\right) .
$$

The same technology gives

$$
\mathcal{R}_{2}(t, \beta)=L r_{1}(2 i \beta)+L^{2} r_{1}(t+i \beta) r_{1}(t-i \beta)-\frac{1}{2} \int d \lambda_{1} d \lambda_{2}\left(\operatorname{Tr}\left(K\left(\lambda_{1}, \lambda_{2}\right) K\left(\lambda_{2}, \lambda_{1}\right)\right)\right) e^{i\left(\lambda_{1}-\lambda_{2}\right) t} e^{-\beta\left(\lambda_{1}+\lambda_{2}\right)},
$$

where 


$$
\begin{aligned}
- & \frac{1}{2} \int d \lambda_{1} d \lambda_{2}\left(\operatorname{Tr}\left(K\left(\lambda_{1}, \lambda_{2}\right) K\left(\lambda_{2}, \lambda_{1}\right)\right)\right) e^{i\left(\lambda_{1}-\lambda_{2}\right) t} e^{-\beta\left(\lambda_{1}+\lambda_{2}\right)} \\
= & -\int d u_{2} e^{-2 \beta u_{2}} \max \left(L \rho\left(u_{2}\right)-\frac{t}{4 \pi}\right. \\
& \left.+\frac{t}{8 \pi} \log \left|1-\frac{t}{2 \pi \rho\left(u_{2}\right) L}\right|, 0\right)
\end{aligned}
$$

Although we did not find an analytic result, one can compute those integrals numerically.

\section{WISHART-LAGUERRE SPECTRAL FORM FACTOR}

\section{A. Random matrix theory review}

In this part, we will consider the Wishart-Laguerre random matrices from the product of square Gaussian ensembles. These Wishart-Laguerre random matrices are squares of standard Gaussian random matrices, which we call a Wishart-Laguerre Unitary Ensemble (LUE), a Wishart-Laguerre Orthogonal Ensemble (LOE), and a Wishart-Laguerre Symplectic Ensemble (LSE), for squares of a GUE, a GOE, and a GSE distribution (For a LSE, we also mean a $2 L \times 2 L$ matrix, while for a $L U E$ and a $L O E$, we mean $L \times L$ matrices). The joint eigenvalue distribution is given by

$$
P(\lambda) \sim|\Delta(\lambda)|^{\tilde{\beta}} \prod_{k=1}^{L} e^{-\frac{\tilde{\beta} L}{4} \lambda_{k}},
$$

where $\tilde{\beta}=1,2,4$ corresponds to LOE, LUE, and LSE ensembles. We are also interested in the $n$-point correlation functions

$$
\rho^{(n)}\left(\lambda_{1}, \ldots, \lambda_{n}\right)=\int d \lambda_{n+1} \ldots d \lambda_{L} P\left(\lambda_{1}, \ldots, \lambda_{L}\right)
$$

The one-point function is the square of the semicircle law in the large $L$ limit, which we could call a Pastur-Marchenko distribution

$$
\rho^{(1)}(\lambda)=\rho(\lambda)=\frac{1}{2 \pi \lambda} \sqrt{\lambda(4-\lambda)}
$$

where now the value of $\lambda$ ranges from 0 to 4 .

We will use the kernels in the large $L$ limit to compute correlation functions and form factors in terms of a box approximation. Similarly, for a LUE, it is a determinant point process, so we could determine the correlation functions as

$$
\rho^{(n)}\left(\lambda_{1}, \ldots, \lambda_{n}\right)=\frac{(L-n) !}{L !} \operatorname{det}\left(K\left(\lambda_{i}, \lambda_{j}\right)\right)_{i, j=1}^{n},
$$

where

$$
K\left(\lambda_{i}, \lambda_{j}\right) \equiv \begin{cases}\frac{\sin \left(L \rho(u) \pi\left(\lambda_{i}-\lambda_{j}\right)\right)}{\pi\left(\lambda_{i}-\lambda_{j}\right)} & \text { for } i \neq j \\ \frac{L}{2 \pi \lambda_{i}} \sqrt{\lambda_{i}\left(4-\lambda_{i}\right)} & \text { for } i=j\end{cases}
$$

with an undetermined constant $u$ from [0,4]. The origination of this constant is from the approximation method, finding an average to put a box in the whole interval $[0,4]$. In the GUE case, we naturally choose $u$ to be 0 because the interval is symmetric in the range $[-2,2]$. However, here in a range with a positive definite eigenvalue, we cannot use such a prescription.

Similarly, for the LOE case, we have

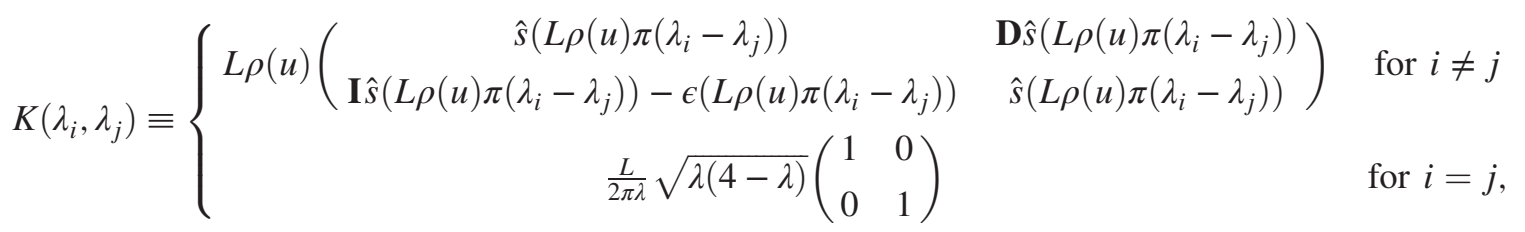

and for the LSE we have

$$
K\left(\lambda_{i}, \lambda_{j}\right) \equiv\left\{\begin{array}{cc}
L \rho(u)\left(\begin{array}{cc}
\hat{s}\left(2 \pi L \rho(u)\left(\lambda_{i}-\lambda_{j}\right)\right) & \mathbf{D} \hat{s}\left(2 \pi L \rho(u)\left(\lambda_{i}-\lambda_{j}\right)\right) \\
\mathbf{I} \hat{s}\left(2 \pi L \rho(u)\left(\lambda_{i}-\lambda_{j}\right)\right) & \hat{s}\left(2 \pi L \rho(u)\left(\lambda_{i}-\lambda_{j}\right)\right)
\end{array}\right) & \text { for } i \neq j \\
\frac{L}{2 \pi \lambda} \sqrt{\lambda(4-\lambda)}\left(\begin{array}{cc}
1 & 0 \\
0 & 1
\end{array}\right) & \text { for } i=j .
\end{array}\right.
$$

The Pfaffian point process will determine the structure of the correlation functions in terms of form factors as

$$
\rho^{(n)}\left(\lambda_{1}, \ldots, \lambda_{n}\right)=\frac{(L-n) !}{L !} \sum_{m \geq 1}^{\cup_{i=1}^{m} S_{i}=\{1, \ldots, n\}}(-1)^{n-m} T_{S_{1}} \ldots T_{S_{m}}
$$


where

$$
T_{n}\left(\lambda_{1}, \ldots, \lambda_{n}\right)=\frac{1}{2 n} \sum_{\sigma} \operatorname{Tr}\left(K\left(\lambda_{\sigma_{1}}, \lambda_{\sigma_{2}}\right) K\left(\lambda_{\sigma_{2}}, \lambda_{\sigma_{3}}\right) \ldots K\left(\lambda_{\sigma_{n}}, \lambda_{\sigma_{1}}\right)\right)
$$

About convolution theorems, in this case, we could obtain

Theorem 4.1 (Convolution formula for LUE): We have

$$
\begin{aligned}
\int & \prod_{i=1}^{m} d \lambda_{i} K\left(\lambda_{1}, \lambda_{2}\right) K\left(\lambda_{2}, \lambda_{3}\right) \ldots K\left(\lambda_{m-1}, \lambda_{m}\right) K\left(\lambda_{m}, \lambda_{1}\right) e^{\sum_{i=1}^{m} i k_{i} \lambda_{i}} \\
& =L r_{3}\left(\sum_{i=1}^{m} k_{i}\right) \int d k g(k) g\left(k+\frac{k_{1}}{2 \pi \alpha_{L}}\right) g\left(k+\frac{k_{2}}{2 \pi \alpha_{L}}\right) \ldots g\left(k+\frac{k_{m-1}}{2 \pi \alpha_{L}}\right),
\end{aligned}
$$

where

$$
\alpha_{L}=L \rho(u)
$$

and

$$
r_{3}(t)=\frac{\sin (t / 2 \rho(u))}{t / 2 \rho(u)}
$$

Theorem 4.2 (Convolution formula for LOE): We have

$$
\begin{aligned}
\int & \prod_{i=1}^{m} d \lambda_{i} K\left(\lambda_{1}, \lambda_{2}\right) K\left(\lambda_{2}, \lambda_{3}\right) \ldots K\left(\lambda_{m-1}, \lambda_{m}\right) K\left(\lambda_{m}, \lambda_{1}\right) e^{i \sum_{i=1}^{m} k_{i} \lambda_{i}} \\
& =L r_{3}\left(\sum_{i=1}^{m} k_{i}\right) \int d k G(k) G\left(k+\frac{k_{1}}{2 \pi \alpha_{L}}\right) G\left(k+\frac{k_{2}}{2 \pi \alpha_{L}}\right) \ldots G\left(k+\frac{k_{m-1}}{2 \pi \alpha_{L}}\right) .
\end{aligned}
$$

Theorem 4.3 (Convolution formula for LSE): We have

$$
\begin{aligned}
\int & \prod_{i=1}^{m} d \lambda_{i} K\left(\lambda_{1}, \lambda_{2}\right) K\left(\lambda_{2}, \lambda_{3}\right) \ldots K\left(\lambda_{m-1}, \lambda_{m}\right) K\left(\lambda_{m}, \lambda_{1}\right) e^{i \sum_{i=1}^{m} k_{i} \lambda_{i}} \\
& =L r_{3}\left(\sum_{i=1}^{m} k_{i}\right) \int d k H(k) H\left(k+\frac{k_{1}}{2 \pi \alpha_{L}}\right) H\left(k+\frac{k_{2}}{2 \pi \alpha_{L}}\right) \ldots H\left(k+\frac{k_{m-1}}{2 \pi \alpha_{L}}\right)
\end{aligned}
$$

Notice that, in order to mimic the delta function, we have to integrate over $\mathbb{R}$ for all variables instead of a bounded range (Similar with the treatment in the Gaussian random matrices). Based on this knowledge, we could start to summarize the results for form factors in the case of Wishart-Laguerre matrices.

\section{B. Result summary}

\section{Two-point form factor}

The two-point form factor has the universal form

$$
\mathcal{R}_{2}=L+L^{2} r_{1}(t) r_{1}^{*}(t)-L r_{2}(t)
$$

where we always have

$$
r_{1}(t)=e^{2 i t}\left(J_{0}(2 t)-i J_{1}(2 t)\right)
$$

For a LUE, we have

$$
r_{2}(t)= \begin{cases}1-\frac{t}{2 \pi L \rho(u)} & \text { for } 0<t<2 \pi L \rho(u) \\ 0 & \text { for } t>2 \pi L \rho(u)\end{cases}
$$

for a LOE, we have 


$$
r_{2}(t)= \begin{cases}1-\frac{t}{\pi \rho(u) L}+\frac{t}{2 \pi \rho(u) L} \log \left(1+\frac{t}{\pi \rho(u) L}\right) & t<2 L \pi \rho(u) \\ -1+\frac{t}{2 \pi \rho(u) L} \log \left(\frac{1+t / \pi \rho(u) L}{t / L \pi(u)-1}\right) & t>2 L \pi \rho(u) .\end{cases}
$$

For a LSE, we have

$$
r_{2}(t)= \begin{cases}1-\frac{t}{4 \pi \rho(u) L}+\frac{t}{8 \pi \rho(u) L} \log \left|1-\frac{t}{2 \pi \rho(u) L}\right| & t<4 L \pi \rho(u) \\ 0 & t>4 L \pi \rho(u) .\end{cases}
$$

\section{Four-point form factor}

The four-point form factor has the universal form

$$
\begin{aligned}
\mathcal{R}_{4} & =L^{4}\left|r_{1}(t)\right|^{4}-2 L^{3} \operatorname{Re}\left(r_{1}^{2}(t)\right) r_{2}(t) r_{3}(2 t)-4 L^{3}\left|r_{1}(t)\right|^{2} r_{2}(t)+2 L^{3} \operatorname{Re}\left(r_{1}(2 t) r_{1}^{* 2}(t)\right)+4 L^{3}\left|r_{1}(t)\right|^{2}+2 L^{2} r_{2}^{2}(t)+L^{2} r_{2}^{2}(t) r_{3}^{2}(2 t) \\
& +6 L^{2} \operatorname{Re}\left(r_{1}(t)\right) r_{3,1}(t) r_{3}(t)+2 L^{2} \operatorname{Re}\left(r_{1}(t)\right) r_{3,2}(t) r_{3}(t)-2 L^{2} \operatorname{Re}\left(r_{1}(2 t) r_{3}(2 t) r_{2}(t)-4 L^{2} \operatorname{Re}\left(r_{1}^{*}(t)\right) r_{3}(t) r_{2}(2 t)\right. \\
& +L^{2}\left|r_{1}(2 t)\right|^{2}-4 L^{2}\left|r_{1}(t)\right|^{2}-4 L^{2} r_{2}(t)+2 L^{2}-6 L r_{4}(t)-L r_{2}(2 t)+4 L r_{3,3}(t)+4 L r_{2}(t)-L,
\end{aligned}
$$

where the dominated term is

$$
\mathcal{R}_{4} \sim L^{4}\left|r_{1}(t)\right|^{4}+2 L^{2} r_{2}^{2}(t)-4 L^{2} r_{2}(t)+2 L^{2}-6 L r_{4}(t)-L r_{2}(2 t)+4 L r_{3,3}(t)+4 L r_{2}(t)-L .
$$

Now, we specify these block functions for different ensembles. For all three ensembles, we still have

$$
r_{1}(t)=e^{2 i t}\left(J_{0}(2 t)-i J_{1}(2 t)\right) \quad r_{3}(t)=\frac{\sin (t / 2 \rho(u))}{t / 2 \rho(u)} .
$$

For a LUE, we have

$$
r_{3,1}(t)=r_{3,2}(t)=r_{3,3}(t / 3)=r_{4}(t / 2)=r_{2}(t)= \begin{cases}1-\frac{t}{2 \pi L \rho(u)} & \text { for } 0<t<2 \pi L \rho(u) \\ 0 & \text { for } t>2 \pi L \rho(u) .\end{cases}
$$

For a LOE, we have

$$
\begin{aligned}
& r_{3,2}(t)=r_{2}(t)= \begin{cases}1-\frac{t}{\pi \rho(u) L}+\frac{t}{2 \pi \rho(u) L} \log \left(1+\frac{t}{\pi \rho(u) L}\right) & t<2 L \pi \rho(u) \\
-1+\frac{t}{2 \pi \rho(u) L} \log \left(\frac{t / \pi \rho(u) L+1}{t / \pi \rho(u)-1}\right) & t>2 L \pi \rho(u)\end{cases} \\
& r_{3,1}(t)= \begin{cases}1-\frac{2 t}{\pi \rho(u) L}+\frac{3 t}{2 \pi \rho(u) L} \log \left(1+\frac{t}{\pi \rho(u) L}\right) & 0<t<\pi \rho(u) L \\
-2+\frac{t}{\pi \rho(u) L}+\frac{3 t}{2 \pi \rho(u) L} \log \left(\frac{1}{2}\left(1+\frac{3}{2 t / \pi \rho(u)-1}\right)\right) & \pi \rho(u) L<t<2 \pi \rho(u) L \\
0 & t>2 \pi \rho(u) L\end{cases} \\
& r_{3,3}(t)= \begin{cases}1-\frac{3 t}{\pi \rho(u) L}+\frac{t}{\pi \rho(u) L} \log \left(1+\frac{t}{\pi \rho(u) L}\right)+\frac{5 t}{4 \pi \rho(u) L} \log \left(1+\frac{2 t}{\pi \rho(u) L}\right) & 0<t<\frac{2}{3} \pi \rho(u) L \\
-2+\frac{3 t}{2 \pi \rho(u) L}+\frac{t}{\pi \rho(u) L} \log \left(\frac{1+t / \pi \rho(u) L}{3 t / \pi \rho(u) L-1}\right)+\frac{5 t}{4 \pi \rho(u) L} \log \left(\frac{1+2 t / \pi \rho(u) L}{3 t / \pi \rho(u) L-1}\right) & \frac{2}{3} \pi \rho(u) L<t<\pi \rho(u) L \\
-1+\frac{t}{2 \pi \rho(u) L}+\frac{5 t}{4 \pi \rho(u) L} \log \left(\frac{1+2 t / \pi \rho(u) L}{3 t / \pi \rho(u) L-1}\right) & \pi \rho(u) L<t<2 \pi \rho(u) L \\
0 & \end{cases} \\
& r_{4}(t)= \begin{cases}1-\frac{7 t}{4 \pi \rho(u) L}+\frac{5 t}{4 \pi \rho(u) L} \log \left(1+\frac{t}{\pi \rho(u) L}\right) & 0<t<\pi \rho(u) L \\
-\frac{3}{2}+\frac{3 t}{4 \pi \rho(u) L}+\frac{5 t}{4 \pi \rho(u) L} \log \left(\frac{1}{2}\left(1+\frac{3}{2 t / \pi \rho(u) L-1}\right)\right) & L<t<2 \pi \rho(u) L \\
0 & t>2 \pi \rho(u) L .\end{cases}
\end{aligned}
$$


For a LSE, we have

$$
\begin{aligned}
r_{3,1} & =r_{4}(t)= \begin{cases}1-\frac{1}{2} \frac{t}{\pi \rho(u) L}+\frac{3}{16} \frac{t}{\pi \rho(u) L} \log \left|\frac{t}{\pi \rho(u) L}-1\right| & t<2 \pi \rho(u) L \\
0 & t>2 \pi \rho(u) L\end{cases} \\
r_{3,2}(t) & =r_{2}(t) \\
r_{3,3} & = \begin{cases}1-\frac{3 t}{4 \pi \rho(u) L}+\frac{t}{32 \pi \rho(u) L} \log \left|\frac{2 t / \pi \rho(u) L-2}{2-t / \pi \rho(u) L}\right|+\frac{9 t}{32 \pi \rho(u) L} \log \left|\frac{3}{2} \frac{t}{\pi \rho(u) L}-1\right| & t<\frac{4}{3} \pi \rho(u) L \\
0 & t>\frac{4}{3} \pi \rho(u) L .\end{cases}
\end{aligned}
$$

\section{Refined two-point form factor}

We will discuss the improvement of the two-point form factor with a finite temperature by the interval method in this section. For a LUE, we have

$$
\begin{aligned}
\mathcal{R}_{2}(t, \beta)= & L r_{1}(2 i \beta)+L^{2} r_{1}(t+i \beta) r_{1}(t-i \beta) \\
& -\int d u_{2} e^{-2 \beta u_{2}} \max \left(L \rho\left(u_{2}\right)-\frac{t}{2 \pi}, 0\right) .
\end{aligned}
$$

When $\beta=0$, the integral is

$$
\begin{aligned}
& \int d u_{2} e^{-2 \beta u_{2}} \max \left(L \rho\left(u_{2}\right)-\frac{t}{2 \pi}, 0\right) \\
& =\frac{L\left(2 \arctan \left(\frac{L-t}{2 t}\right)+\pi\right)}{2 \pi},
\end{aligned}
$$

so we get

$$
\mathcal{R}_{2}(t)=L^{2}\left|r_{1}(t)\right|^{2}+\frac{L\left(\pi-2 \arctan \left(\frac{L}{2 t}-\frac{t}{2 L}\right)\right)}{2 \pi} .
$$

The early time expansion of the connected piece gives

$$
R_{2}^{\mathrm{conn}}(t) \approx \frac{2 t}{\pi}+\mathcal{O}\left(t^{3}\right)
$$

which means that in the box approximation, we could approximately set

$$
\rho(u)=\frac{1}{4} \Rightarrow u=\frac{16}{4+\pi^{2}},
$$

and then it could approximately capture the form factor dynamics.

For a LOE, we have

$$
\begin{aligned}
\mathcal{R}_{2}(t, \beta)= & L r_{1}(2 i \beta)+L^{2} r_{1}(t+i \beta) r_{1}(t-i \beta) \\
& -\int d u_{2} e^{-2 \beta u_{2}} \max \left(L \rho\left(u_{2}\right)-\frac{t}{\pi}+\frac{t}{2 \pi} \log \left(1+\frac{t}{\pi \rho\left(u_{2}\right) L}\right),-L \rho\left(u_{2}\right)+\frac{t}{2 \pi} \log \left(\frac{1+\frac{t}{\pi \rho\left(u_{2}\right) L}}{\frac{t}{\pi \rho\left(u_{2}\right) L}-1}\right)\right)
\end{aligned}
$$

This time, in the $\beta=0$ case, the expansion gives

$$
\mathcal{R}_{2}^{\text {conn }}(t) \approx \frac{4 t}{\pi}+\mathcal{O}\left(t^{3}\right)
$$

but we still have

$$
\rho(u)=\frac{1}{4} \Rightarrow u=\frac{16}{4+\pi^{2}} .
$$

For a LSE case, we have

$$
\begin{aligned}
\mathcal{R}_{2}(t, \beta)= & L r_{1}(2 i \beta)+L^{2} r_{1}(t+i \beta) r_{1}(t-i \beta) \\
& -\int d u_{2} e^{-2 \beta u_{2}} \max \left(L \rho\left(u_{2}\right)-\frac{t}{4 \pi}\right. \\
& \left.+\frac{t}{8 \pi} \log \left|1-\frac{t}{2 \pi \rho\left(u_{2}\right) L}\right|, 0\right),
\end{aligned}
$$

where in the $\beta=0$ case, the expansion gives

$$
\mathcal{R}_{2}^{\operatorname{conn}}(t) \approx \frac{t}{\pi}+\mathcal{O}\left(t^{3}\right)
$$

and the solution of $u$ is still the same

$$
\rho(u)=\frac{1}{4} \Rightarrow u=\frac{16}{4+\pi^{2}} .
$$

\section{FIGURES}

We obtain numerous analytic results in the previous sections. In this section, we will try to plot some of those results. 

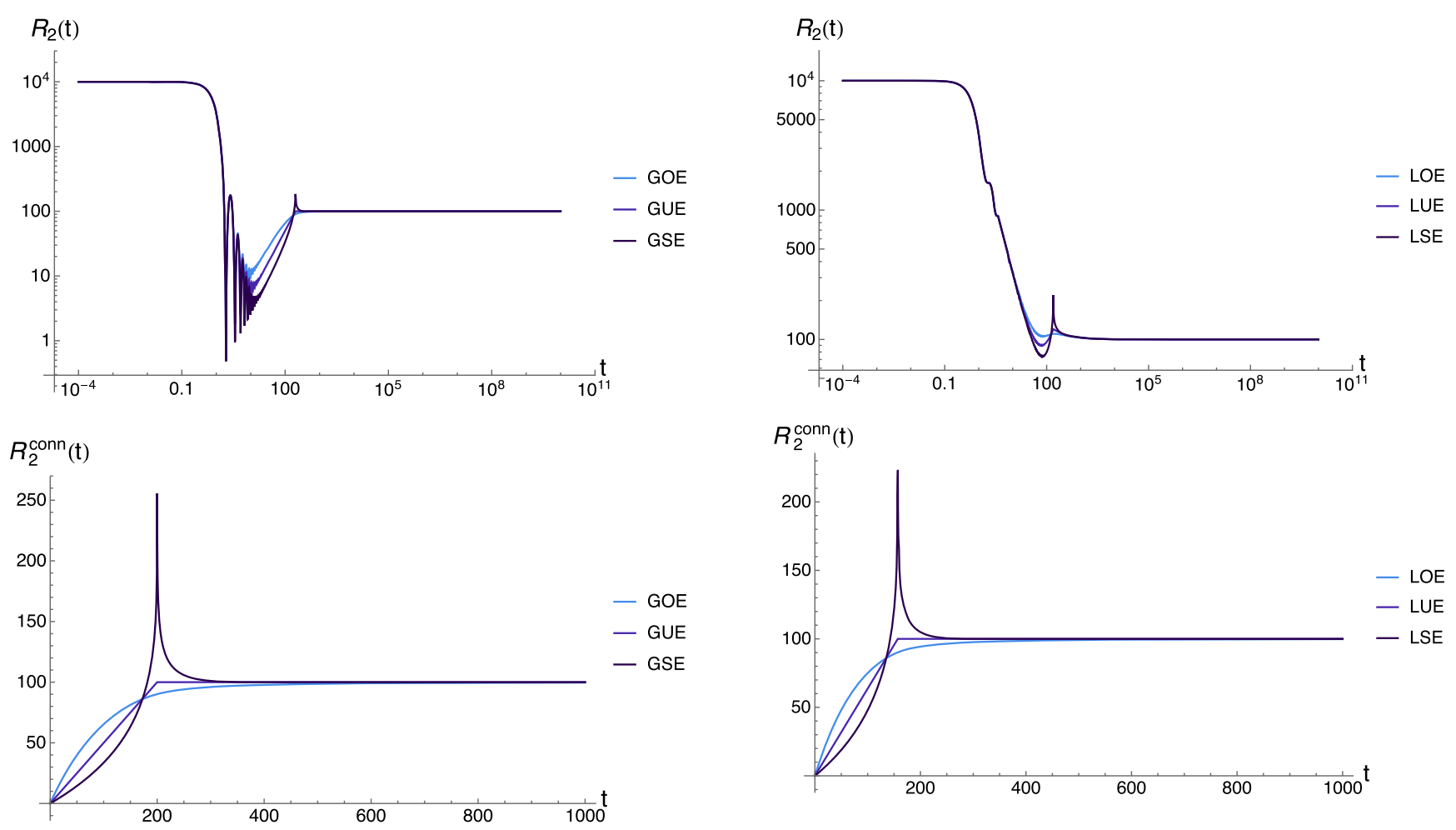

FIG. 1. GOE, GUE, GSE two-point form factors $\mathcal{R}_{2}(t)$ with box cutoff and infinite temperature. We choose $L=100$. Up: full form factor; Down: connected form factor.

In Fig. 1 we are describing the two-point spectral form factors in Gaussian ensembles. One could observe that the main difference among the three ensembles is the behavior around the plateau time. We have a smooth corner for the GOE, a kink for the GUE and a sharp peak for a GSE. These features are universal also for because of different sine kernels.

Figure 2 shows a similar behavior for the four-point form factor $\mathcal{R}_{4}$.

We plot similar figures for Wishart-Laguerre ensembles in Figs. 3 and 4. The main difference is the decay rate in the relatively early time from $r_{1}$. Expanding $r_{1}(t)$ we get $r^{-3 / 2}$

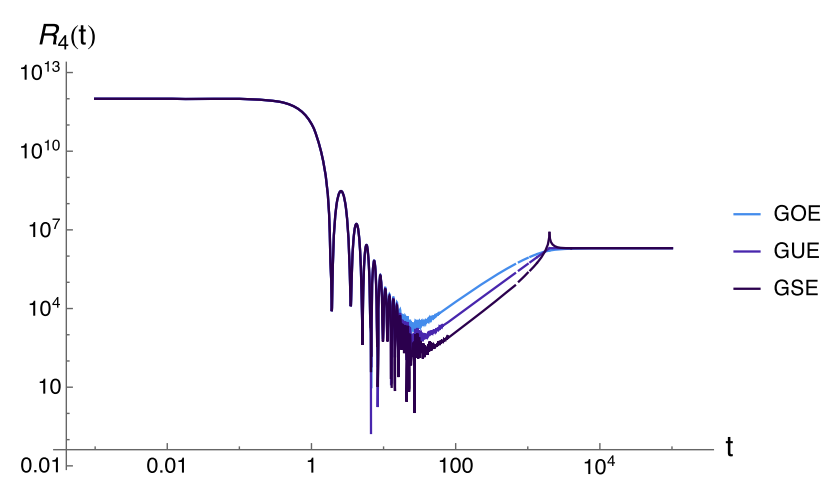

FIG. 2. GOE, GUE, GSE four-point form factors $\mathcal{R}_{4}(t)$ with a box cutoff and infinite temperature. We choose $L=1000$.

FIG. 3. LOE, LUE, LSE two-point form factors $\mathcal{R}_{2}(t)$ with a box cutoff and infinite temperature. We choose $L=100$. Up: full form factor; Down: connected form factor.

for Gaussian ensembles and $r^{-1 / 2}$ for Wishart-Laguerre ensembles. A direct comparison is displayed in Fig. 5.

There will be an interesting comparison showing the improvement from the box approximation to the refined form factors. Thus, we give Fig. 6 for the connected piece of the GUE. The box approximation gives a linear result from $(0,0)$ to $(2 L, L)$. The plateau value $L$ and the plateau time $2 L$ are both correct. However, the correct slope, should be $2 / \pi$ instead of $1 / 2$. Thus, one may consider the Taylor expansion (a naive approximation only chooses the slope, namely the derivative, at a relatively early time) to capture the correct slope. Maintaining the correct slope

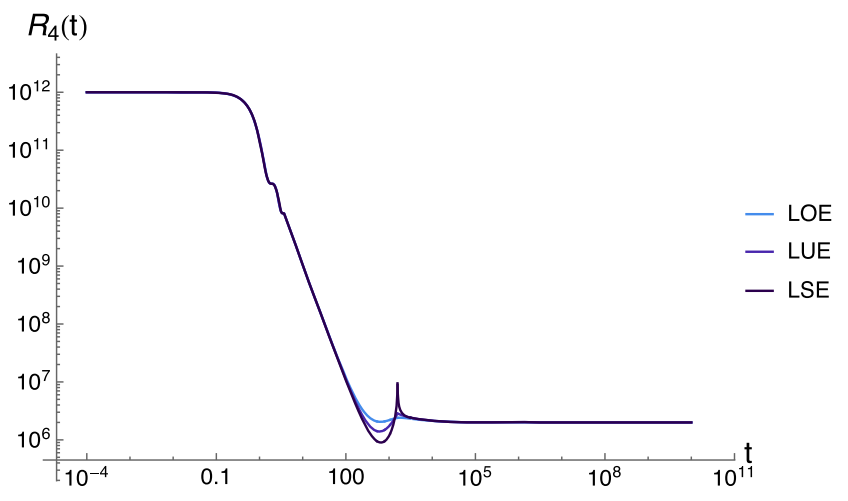

FIG. 4. LOE, LUE, LSE four point form factors $\mathcal{R}_{4}(t)$ with a box cutoff and infinite temperature. We choose $L=1000$. 

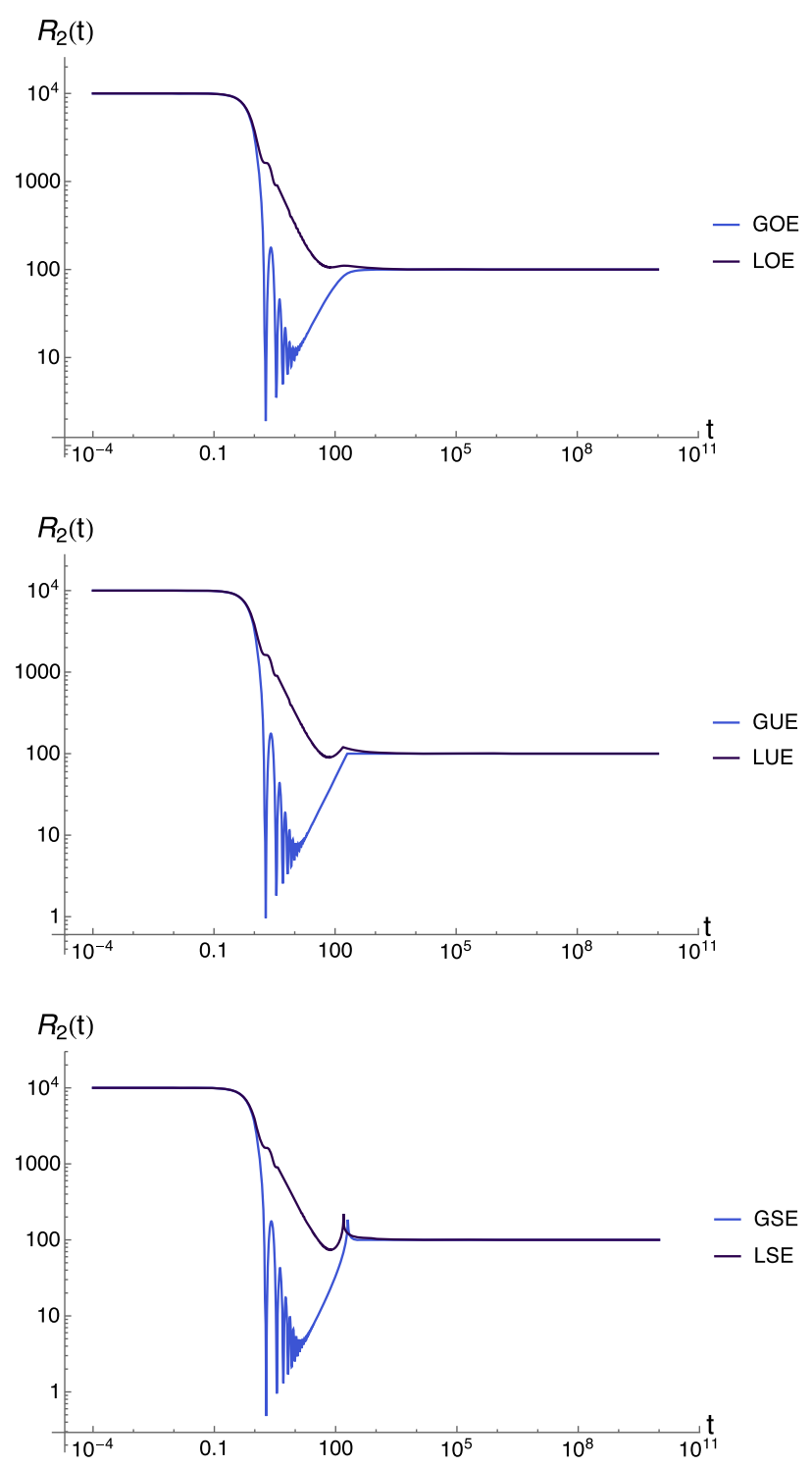

FIG. 5. A direct comparison between Gaussian ensembles and Wishart-Laguerre ensembles in terms of two-point form factor $\mathcal{R}_{2}(t)$ with a box cutoff and infinite temperature. We choose $L=100$. Up GOE/LUE; Middle: GUE/LUE; Down: GSE/LSE.

and the plateau value, the plateau time is inaccurate. Thus, a refinement will be to consider a nonlinear improvement, which is given by our previous small interval integrals over the short distance kernel. The situation is precisely described in Fig. 6.

One can generalize this analysis to other Gaussian ensembles, and also Wishart-Laguerre ensembles, which are described in Figs. 7 and 8. One can notice that there is an interesting observation, where the kinky behavior near the plateau time for GSEs and LSEs is suppressed, which causes a deviation between the box approximation and the small interval approximation.

One can also take a look at the connected finite temperature predictions from the refined kernel. We give them in

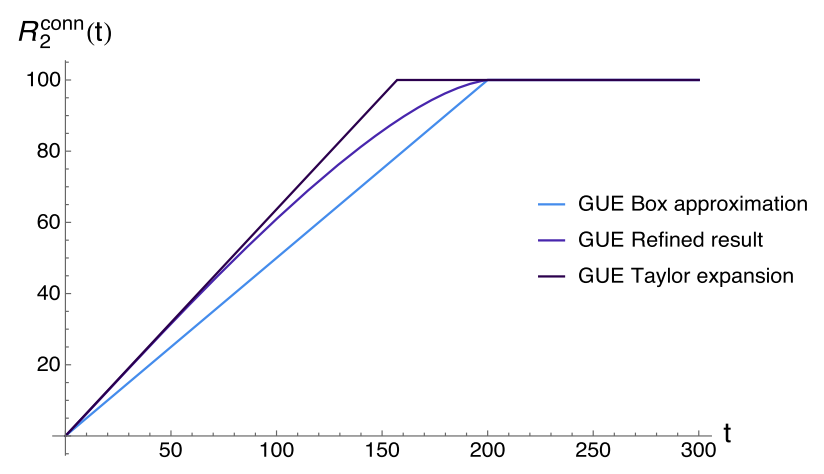

FIG. 6. GUE connected form factor $\mathcal{R}_{2}^{\text {conn }}(t)$ with different approximations in the infinite temperature. We choose $L=100$.

Figs. 9 and 10 for Gaussian and Wishart-Laguerre ensembles separately.

\section{APPLICATIONS}

The spectral form factors of random matrix theory in the standard ensembles have wide applications in many areas of late time quantum chaos. In this section, we will review and summarize some of the applications with recent interest.

\section{A. SYK-like models and classifications}

One direct application of the random matrix theory form factor results will be matching the qualitative and
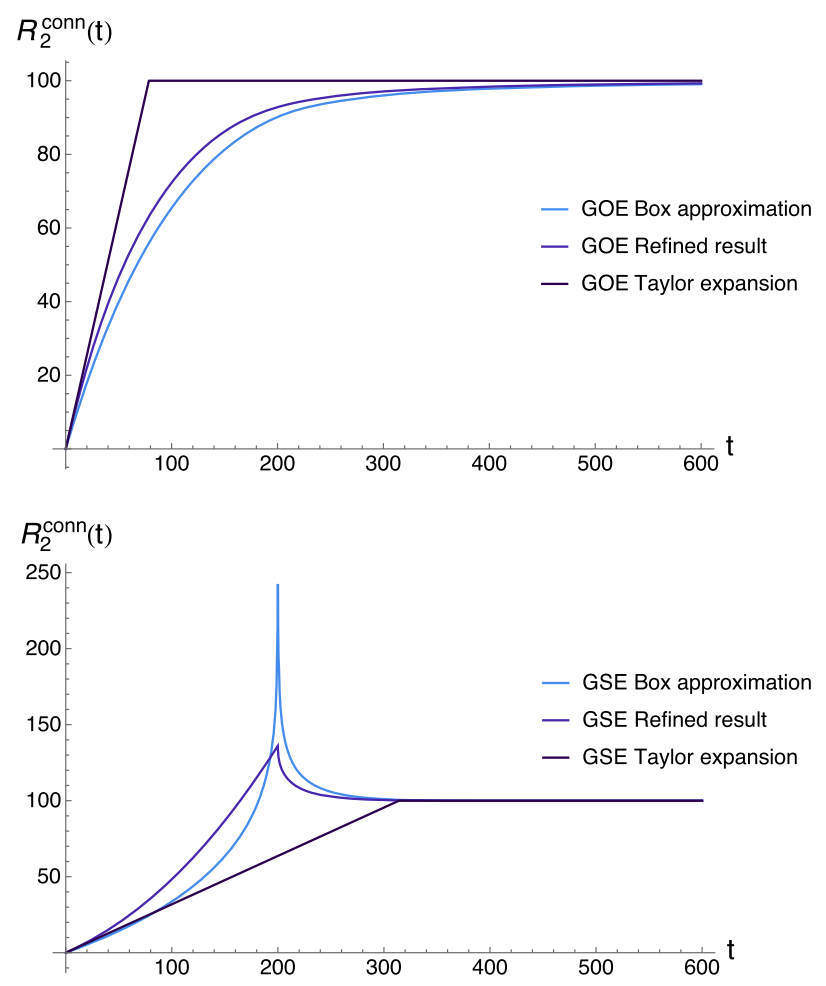

FIG. 7. GOE(up) and GSE(down) connected form factor $\mathcal{R}_{2}^{\text {conn }}(t)$ with different approximations in the infinite temperature. We choose $L=100$. 


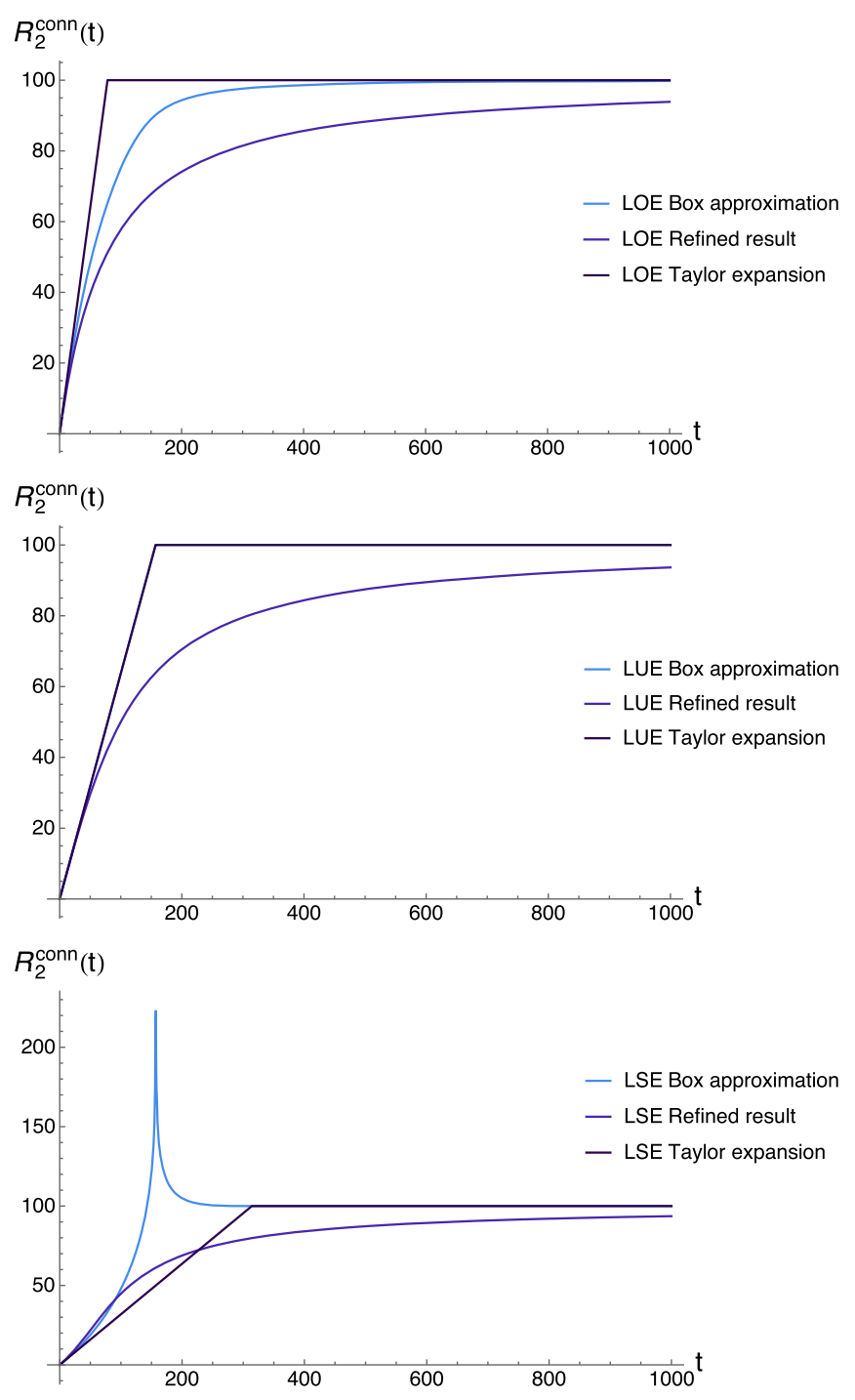

FIG. 8. LOE(up), LUE(middle) and LSE(down) connected form factor $\mathcal{R}_{2}^{\text {conn }}(t)$ with different approximations in the infinite temperature. We choose $L=100$. For a LUE case, by choosing $u$ in the box approximation, the Taylor expansion curve and the box approximation curve are the same, so two of three curves are the same for the figure in the middle.

quantitative behaviors of the spectral form factor of the SYK model. In the Majorana SYK model, there exists an eightfold classification of random matrix theory, with respect to the number of Majorana fermions $N$ [20-22]. The classification is $N \bmod 8=0$ for a GOE, $N \bmod 8=4$ for a GSE, and $N \bmod 8=2,6$ for a GUE. The matching is identified for level statistics and the degeneracies.

One can also study the spectral form factor of the theory. One can show that for the simplest form factor $\mathcal{R}_{2}$, it could also be identified as the combination of the analyticcontinued partition function, $\langle Z(\beta+i t) Z(\beta-i t)\rangle \sim$ $\mathcal{R}_{2}(t, \beta)$. From the SYK model, one can read off the dip time, the plateau time, and the ramp slope. These quantities could be qualitatively and quantitatively checked by

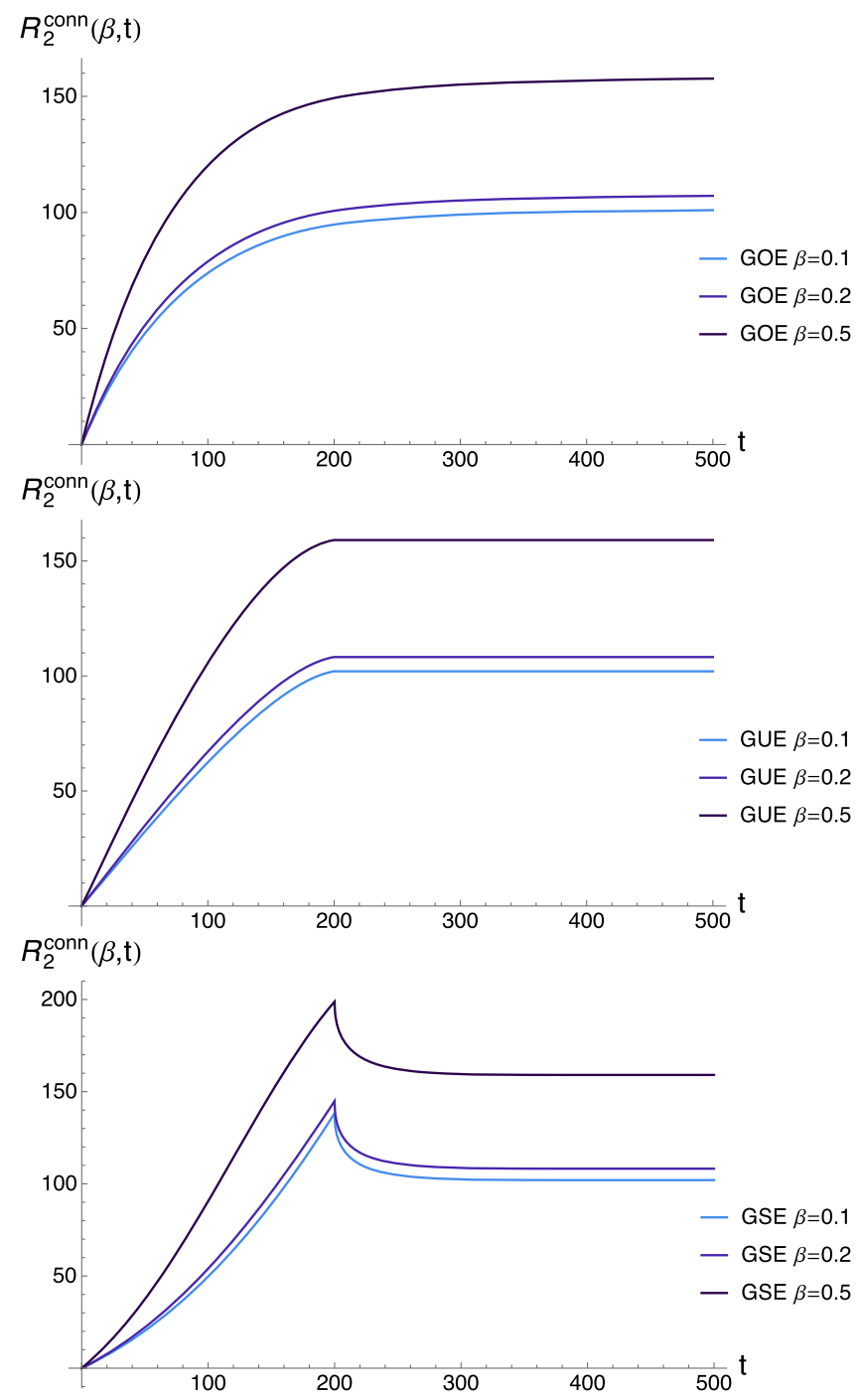

FIG. 9. GOE(up), GUE(middle) and GSE(down) connected form factor $\mathcal{R}_{2}^{\text {conn }}(t, \beta)$ for finite temperatures. We choose $L=100$.

numerical simulations and match with the corresponding random matrix theory prediction [22].

One can observe the eightfold classification of the random matrix theory prediction in the SYK model by observing features of the plots. For instance, one can observe a smooth corner for a GOE, a kink for a GUE, and a sharp peak for a GSE around the time scale of the plateau time. These features will show a clear threefold classification of the SYK model in terms of spectral form factors, and they could be read off from numerical investigations [22].

These ideas could also be generalized to supersymmetric SYK models. In supersymmetric models, one would expect a disordered supercharge, $Q$, and a Hamiltonian, $H \sim Q^{2}$. Thus, if $Q$ is from some Gaussian-like statistics, the result of the squared Gaussian distribution will be the WishartLaguerre-type ensembles. The classification is discussed in 


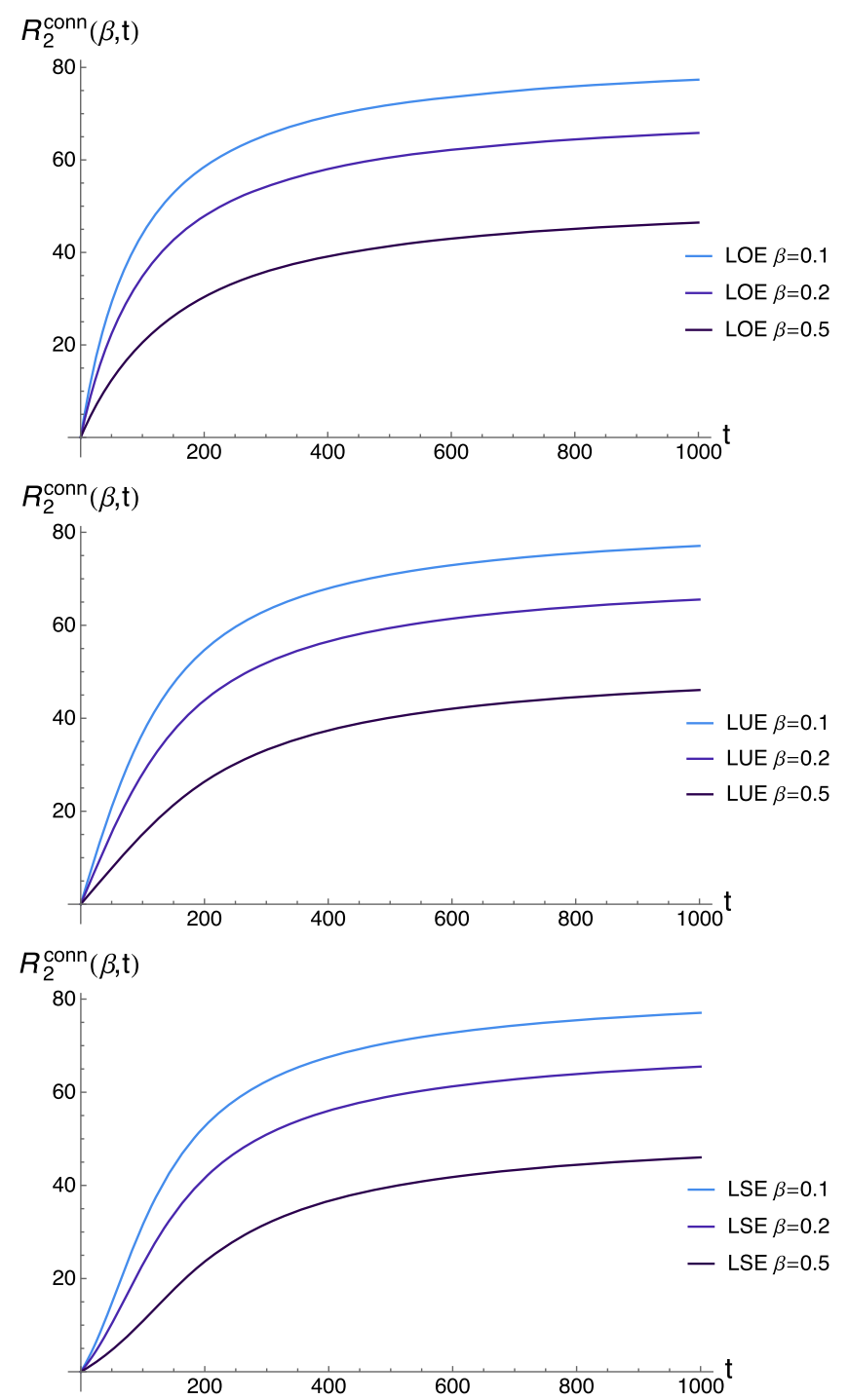

FIG. 10. LOE(up), LUE(middle) and LSE(down) connected form factor $\mathcal{R}_{2}^{\text {conn }}(t, \beta)$ for finite temperatures. We choose $L=100$.

[26,27]. For the simplest case $(\mathcal{N}=1$ supersymmetrization and four-point coupling), the eightfold classification is modified by $N \bmod 8=0,6$ for a LOE, and $N \bmod 8=2$, 4 for a LSE.

An early look of the Wishart-Laguerre ensemble's spectral form factor and a connection to the supersymmetric SYK model are discussed in [26-28], where the features are clearly different from the Gaussian ensembles. One of the main differences is the early time behavior of the disconnected spectral form factor $\mathcal{R}_{2}$, which could be obtained from the $r_{1}(t)$ function that we discussed before in these two different ensembles. In Gaussian ensembles, we have $r_{1}(t) \sim 1 / t^{3 / 2}$, while for Wishart-Laguerre ensembles, we have $r_{1}(t) \sim 1 / t^{1 / 2}$. These facts could match with predictions in the SYK model, and they could be obtained by the one-loop Schwarzian action [22,28,29]. Moreover, one can also match the kinky and smooth behavior around the edge of the plateau from the numerics of the supersymmetric SYK model [26].

\section{B. Out-of-time-ordered correlation functions}

The spectral form factor of random matrix theory could be related to out-of-time-ordered correlators of the physical models in an interesting way. Here, we will discuss the unitary invariance case, where disordered physical models are invariant, or nearly invariant, under a unitary transformation. For Gaussian and Wishart-Laguerre disordered models, one may predict them using a GUE or a LUE.

In this case [23,28], for operators $A$ and $B$, one can compute the two-point correlator as

$$
\langle A(0) B(t)\rangle=\langle A\rangle\langle B\rangle+\frac{\mathcal{R}_{2}(t)-1}{L^{2}-1}\langle\langle A B\rangle\rangle,
$$

where

$$
\langle\langle A B\rangle\rangle=\langle A B\rangle-\langle A\rangle\langle B\rangle
$$

Moreover, if $A$ and $B$ are nonidentity Pauli operators, we have

$$
\langle A(0) B(t)\rangle= \begin{cases}\frac{\mathcal{R}_{2}(t)-1}{L^{2}-1} & A=B \\ 0 & A \neq B .\end{cases}
$$

Thus, if $\mathcal{R}_{2}(t) \gg 1$, we have

$$
\langle A(0) B(t)\rangle \sim \frac{\mathcal{R}_{2}(t)}{L^{2}} .
$$

Similarly, one can generalize those relations towards fourpoint or higher-point functions. For four-point functions, assuming nonidentity Pauli operators $A, B, C, D$ with the relation $A B C D=I$, we have

$$
\langle A(0) B(t) C(0) D(t)\rangle \sim \frac{\mathcal{R}_{4}(t)}{L^{4}},
$$

another important object in quantum information, which will show the averaged behavior of the out-of-time-ordered correlation function, is called the frame potential. For a given ensemble $\mathcal{E}$, the $k$ th order frame potential is defined by

$$
\mathcal{F}_{\mathcal{E}}^{(k)}=\int_{U, V \in \mathcal{E}} d U d V\left|\operatorname{Tr}\left(U V^{\dagger}\right)\right|^{k}
$$

One can define $\mathcal{E}$ to be generated by the disordered Hamiltonian $H$ with a fixed time $t, \mathcal{E}_{t}=\left\{e^{i H t}, H \in\right.$ disorder ensemble $\}$, so $\mathcal{F}$ is identified as a functional of 
a disordered system and a fixed time $t$. In [30], one can show that the frame potential is equal to the average of out-of-time-ordered correlators, where the average is over the Pauli group.

One can compute the relationship between the spectral form factor and the frame potential in random matrix theory $[23,28]$. For instance, in the two-point case, we have

$$
\mathcal{F}^{(1)}(t)=\frac{\mathcal{R}_{2}^{2}(t)+L^{2}-2 \mathcal{R}_{2}(t)}{L^{2} \cdot-1}
$$

One can generate these types of connections to higher-point cases and finite temperatures.

\section{Page states}

A connection between Wishart-Laguerre ensembles and the Page states is used to be a modified criterion for quantum chaos in terms of wave functions [24]. The Page state, or alternatively called the random pure state, is defined as the following wave function in the Hilbert space $\mathcal{H}=\mathcal{H}_{A} \otimes \mathcal{H}_{B}$,

$$
|\psi\rangle=\sum_{a=1}^{N_{A}} \sum_{b=1}^{N_{B}} X_{a b}\left|\psi_{A}^{a}\right\rangle\left|\psi_{B}^{b}\right\rangle,
$$

where $X_{a b}$ is the element of the random matrix with the volume $N_{A} \times N_{B}$, and one could fix the scaling by the normalization condition of the wave function. One can assume that this matrix $X$ is a Gaussian $N_{A} \times N_{B}$ matrix. Thus, the reduced density matrix, when tracing out the system $B$, is given by

$$
\rho_{A} \sim X X^{\dagger}
$$

for subsystem $A$. Now one can consider diagonalization of $\rho_{A}$ and compute the spectral form factor of it. Because of the squaring structure $X X^{\dagger}$, the density matrix $\rho_{A}$ will be a Wishart-Laguerre random matrix. (Here, $N_{A}$ and $N_{B}$ are kept, in general, while in our previous computation, we choose the specific case where $N_{A}=N_{B}$. When $N_{A} \neq N_{B}$, the result will be different, but some generic features are similar with the equal case.)

This feature will appear in some real chaotic physical systems. In [24], it is claimed that in splitting the qubits of the real chaotic system, one would expect that the reduced density matrix, or namely, the entanglement Hamiltonian, will show a similar universal spectral correlation and will match the prediction of Wishart-Laguerre ensemble when considering time evolution. This phenomenon is verified in the context of the Floquet system and quantum Ising model.

\section{CONCLUSION AND DISCUSSION}

In this paper, we investigate spectral form factors in detail, to establish a generic framework on the computational technology. We hope that those technologies will give a systematic description of spectral form factors that are used in the field of quantum chaos, and will benefit people studying the connection between random matrix theory and notions of quantum chaos, quantum information, and black holes, etc.

We will highlight some of the points of this paper with the following:

(i) Traditional literature (for instance [7]) refers to the $n$-point spectral form factor as the Fourier transformation of $n-1$ eigenvalue variables. To transform the last variable, one obtains a delta function in the infinite $L$ limit. For a finite but large $L$, one has to invent some regularization technologies. In this paper, we systematically describe the notion of the box approximation as a concrete way to realize the cutoff, and we apply it to multiple ensembles. We also show, in the Gaussian ensemble two-point form factor context, that the approximation beyond the box cutoff must be related to nonlinear physics for the Fourier transformation of the sine kernel.

(ii) We seriously consider how to use the short distance kernel to give a precise prediction for the two-point form factor with infinite and finite temperatures. Inspired by the treatment from $[22,24]$, we obtain an analytic nonlinear connected two-point form factor beyond the linear approximation in the GUE and LUE, and we show the formal and numerical results for the rest of the cases.

(iii) Based on existing infinite $L$ mathematical algorithms, we illustrate some theorems that could be used to compute higher-point form factors for finite but large $L$ for multiple ensembles. We compute the four-point form factors for them as examples.

We hope that this research will shed light on the possibilities of the following directions:

(i) More general ensembles. Although the situations that are considered are already pretty general, mathematicians and mathematical physicists have a list of more general ensembles. It will be interesting to consider generalized classifications and related ensembles, and compute the spectral form factors of them.

(ii) More physical applications. One may consider applying those form factors to some other chaotic quantum systems and more black hole thought experiments, such as chaotic spin chains or quantum circuits.

(iii) Diving deeper into physical meaning of the nonlinearity in the connected two-point form factor. The breakdown of the naive box cutoff, namely the prediction beyond the linear approximation of the Fourier transformation of the sine kernel, might be connected to some physics of thermalization and, moreover, holography and gravity [25]. 


\section{ACKNOWLEDGMENTS}

I deeply thank Beni Yoshida for his warm and supportive guidance and Jordan Cotler for nice communications. J. L. is supported in part by the Institute for Quantum Information and Matter (IQIM), an NSF Physics
Frontiers Center (NSF Grant No. PHY-1125565) with support from the Gordon and Betty Moore Foundation (GBMF-2644), and by the Walter Burke Institute for theoretical physics.
[1] E. Wigner, Ann. Math. 62, 548 (1955).

[2] F. Dyson, J. Math. Phys. (N.Y.) 3, 140 (1962).

[3] O. Bohigas, M. J. Giannoni, and C. Schmit, Phys. Rev. Lett. 52, 1 (1984).

[4] M. R. Zirnbauer, J. Math. Phys. (N.Y.) 37, 4986 (1996).

[5] A. Altland and M. R. Zirnbauer, Phys. Rev. B 55, 1142 (1997).

[6] T. Tao, Topics in Random Matrix Theory (American Mathematical Society, Providence, 2012).

[7] M. Mehta, Random Matrices, 2nd ed (Academic Press, 1990).

[8] E. Brezin and S. Hikami, Random Matrix Theory with an External Source (Springer, Singapore, 2017).

[9] P. Deift and D. Gioev, Random Matrix Theory: Invariant Ensembles and Universality (American Mathematical Society, Providence, 2009).

[10] L. Pastur, M. Shcherbina, and M. Shcherbina, Eigenvalue Distribution of Large Random Matrices (American Mathematical Society, Providence, 2011).

[11] P. Hayden and J. Preskill, J. High Energy Phys. 09 (2007) 120.

[12] Y. Sekino and L. Susskind, J. High Energy Phys. 10 (2008) 065.

[13] N. Lashkari, D. Stanford, M. Hastings, T. Osborne, and P. Hayden, J. High Energy Phys. 04 (2013) 022.

[14] S. H. Shenker and D. Stanford, J. High Energy Phys. 03 (2014) 067.

[15] S. H. Shenker and D. Stanford, J. High Energy Phys. 05 (2015) 132.

[16] J. Maldacena, S. H. Shenker, and D. Stanford, J. High Energy Phys. 08 (2016) 106.
[17] S. Sachdev and J. Ye, Phys. Rev. Lett. 70, 3339 (1993).

[18] A. Kitaev, Hidden correlations in the hawking radiation and thermal noise, in Proceedings of the Fundamental Physics Prize Symposium, 2014 and at the KITP, 2015.

[19] J. Maldacena and D. Stanford, Phys. Rev. D 94, 106002 (2016).

[20] Y.Z. You, A. W. W. Ludwig, and C. Xu, Phys. Rev. B 95, 115150 (2017).

[21] A. M. Garcia-Garcia and J. J. M. Verbaarschot, Phys. Rev. D 94, 126010 (2016).

[22] J. S. Cotler, G. Gur-Ari, M. Hanada, J. Polchinski, P. Saad, S. H. Shenker, D. Stanford, A. Streicher, and M. Tezuka, J. High Energy Phys. 05 (2017) 118.

[23] J. Cotler, N. Hunter-Jones, J. Liu, and B. Yoshida, J. High Energy Phys. 11 (2017) 048.

[24] X. Chen and A. W. W. Ludwig, Phys. Rev. B 98, 064309 (2018).

[25] H. Gharibyan, M. Hanada, S. H. Shenker, and M. Tezuka, J. High Energy Phys. 07 (2018) 124.

[26] T. Li, J. Liu, Y. Xin, and Y. Zhou, J. High Energy Phys. 06 (2017) 111.

[27] T. Kanazawa and T. Wettig, J. High Energy Phys. 09 (2017) 050.

[28] N. Hunter-Jones and J. Liu, J. High Energy Phys. 05 (2018) 202.

[29] D. Stanford and E. Witten, J. High Energy Phys. 10 (2017) 008.

[30] D. A. Roberts and B. Yoshida, J. High Energy Phys. 04 (2017) 121. 